ARTICLE

\title{
The anti-malarial atovaquone increases radiosensitivity by alleviating tumour hypoxia
}

Thomas M. Ashton', Emmanouil Fokas, Leoni A. Kunz-Schughart1,2, Lisa K. Folkes', Selvakumar Anbalagan, Melanie Huether ${ }^{2}$, Catherine J. Kelly ${ }^{1}$, Giacomo Pirovano ${ }^{1}$, Francesca M. Buffa ${ }^{1}$, Ester M. Hammond ${ }^{1}$, Michael Stratford ${ }^{1}$, Ruth J. Muschel ${ }^{1}$, Geoff S. Higgins ${ }^{1, \star} \&$ William Gillies McKenna ${ }^{1, \star}$

Tumour hypoxia renders cancer cells resistant to cancer therapy, resulting in markedly worse clinical outcomes. To find clinical candidate compounds that reduce hypoxia in tumours, we conduct a high-throughput screen for oxygen consumption rate (OCR) reduction and identify a number of drugs with this property. For this study we focus on the anti-malarial, atovaquone. Atovaquone rapidly decreases the OCR by more than $80 \%$ in a wide range of cancer cell lines at pharmacological concentrations. In addition, atovaquone eradicates hypoxia in FaDu, HCT116 and H1299 spheroids. Similarly, it reduces hypoxia in FaDu and HCT116 xenografts in nude mice, and causes a significant tumour growth delay when combined with radiation. Atovaquone is a ubiquinone analogue, and decreases the OCR by inhibiting mitochondrial complex III. We are now undertaking clinical studies to assess whether atovaquone reduces tumour hypoxia in patients, thereby increasing the efficacy of radiotherapy.

\footnotetext{
${ }^{1}$ CRUK/MRC Oxford Institute for Radiation Oncology, Old Road Campus Research Building, Roosevelt Drive, Oxford OX3 7DQ, UK. ${ }^{2}$ OncoRay - National Center for Radiation Research in Oncology, Faculty of Medicine and University Hospital Carl Gustav Carus, TU Dresden, and Helmholtz-Zentrum DresdenRossendorf, Institute of Radiooncology, Dresden, P.O. Box 41, 01307, Germany. * These authors contributed equally to this work. Correspondence and requests for materials should be addressed to G.S.H. (email: geoffrey.higgins@oncology.ox.ac.uk) or to W.G.M. (email: gillies.mckenna@gmail.com).
} 
S olid malignancies frequently have regions of hypoxia ${ }^{1}$. Many studies have focused on the abnormal tumour vasculature as a determinant of tumour hypoxia. However, tumour oxygenation reflects the balance between delivery and consumption, and because tumour cells have a high level of oxygen consumption, and reduced delivery of oxygen to the tumour due to abnormal vasculature, both components play a role ${ }^{2}$. Hypoxia is associated with poor clinical outcomes due to both local recurrence and an increased probability of metastasis ${ }^{3}$. Gray and his colleagues in the 1950s were the first to suggest that hypoxia would affect the outcome of radiotherapy because hypoxic tumour cells are up to three times more radioresistant than normoxic tumour cells $s^{1,3,4}$. This radioresistance is due to the absence of the oxygen enhancement effect, which is a result of the direct physicochemical reaction of oxygen with the broken ends of the DNA strands that result from radiation, creating stable organic peroxides that are more difficult for the cell to repair.

Vascular remodelling has been used previously as a strategy to relieve tumour hypoxia and improve radiation response, and it has been demonstrated that a number of signal transduction inhibitors have such an effect ${ }^{5}$. A possible alternative strategy to reduce tumour hypoxia, and therefore to increase radiosensitivity, is to reduce the cellular oxygen consumption rate (OCR). Reduction of the OCR in three-dimensional (3D) multicellular tumour spheroids causes a decrease in the volume of the central region of hypoxia by increasing the availability of oxygen throughout the spheroid ${ }^{6-8}$. These studies in spheroids demonstrate that reducing the OCR can alleviate hypoxia in an avascular system. Mathematical modelling suggests that a $30 \%$ decrease in the OCR would even abolish severe tumour hypoxia, and that this could be a more effective approach to reduce hypoxia than attempts at elevating blood flow or increasing the oxygen levels in blood ${ }^{8}$. An additional advantage of assessing the OCR is that it is amenable to high-throughput screening, which vascular remodelling is not. Drugs that reduce the OCR have been identified previously by screens designed to identify inhibitors of the HIF-1 pathway ${ }^{9,10}$, but to our knowledge, no high-throughput screens designed to identify novel inhibitors of the OCR in cancer cells have been published.

The anti-diabetic, metformin, is the only FDA-approved drug that has been shown to reduce both the OCR and tumour hypoxia, and its mechanism of action is inhibition of mitochondrial complex I (refs 11,12). However, at pharmacological concentrations of metformin there is a reduction in the OCR of only $10-20 \%$, suggesting that compounds with a more significant effect on the OCR may have a more profound effect on tumour hypoxia ${ }^{12}$. Thus there is a need to identify more drugs that alleviate tumour hypoxia by reducing the OCR.

To identify drugs that decrease the OCR of cancer cells and could be used as modifiers of tumour hypoxia in a clinical setting, we screen a library of 1,697 FDA-approved compounds. This screen identifies atovaquone, a drug primarily used to treat malaria and pneumocystis pneumonia. Atovaquone reduces the OCR by inhibition of mitochondrial complex III (the cytochrome $b c_{1}$ complex) at pharmacologically achievable concentrations, alleviates hypoxia both in spheroids and in xenografted tumours, and causes a significant tumour growth delay in combination with radiation.

\section{Results}

High-throughput screening to assess oxygen consumption. To identify compounds that reduce the OCR, FaDu hypopharyngeal carcinoma cells were incubated with 1,697 FDA-approved compounds at 2 or $10 \mu \mathrm{M}$ for $24 \mathrm{~h}$ (Fig. 1a). The growth medium was replaced with an assay medium containing $5 \mathrm{mM}$ galactose, $5 \mathrm{mM}$ pyruvate and $4 \mathrm{mM}$ glutamine. Growth in galactose promotes oxidative phosphorylation ${ }^{13}$. The mitochondrialspecific OCR was determined by measuring basal OCR and subtracting the OCR measured following injection of $2 \mu \mathrm{M}$ antimycin A to inhibit mitochondrial respiration. The OCR measurements were corrected for cell number using the relative Hoechst fluorescence of the cells fixed immediately after the assay. Compounds that caused a reduction in cell number of more than $66 \%$ compared with the DMSO control wells for each plate were excluded. The compounds with the top 130 rank products were chosen for the secondary screen (Supplementary Data 1). The secondary screen was conducted using the same protocol as the primary screen, except $\mathrm{FaDu}$ cells were incubated with the compounds at $80 \mathrm{nM}, 400 \mathrm{nM}, 2 \mu \mathrm{M}$ or $10 \mu \mathrm{M}$, and the assay medium contained $5 \mathrm{mM}$ glucose instead of $5 \mathrm{mM}$ galactose to more closely mimic the physiological setting (Fig. $1 \mathrm{~b}$ and Supplementary Data 1). The highest ranked compounds that caused a decrease in the OCR in FaDu cells at $10 \mu \mathrm{M}$ and are approved for systemic use in humans are shown in Table 1.

Compounds were subsequently excluded from further study based on a range of criteria (Supplementary Table 1): Known in vivo hypoxia modifiers or radiosensitisers such as acriflavinium ${ }^{14}$, compounds with an unsuitable safety profile such as emetine $^{15}$, anti-helminths with poor bioavailability such as pyrvinium $^{16}$, and compounds for which the maximum achievable plasma concentration in patients is lower than the concentration required to cause a significant reduction in the OCR, such as dactinomycin ${ }^{17}$. The anti-malarial, atovaquone was selected for further evaluation, because it is not known to reduce hypoxia in vivo, it has an excellent safety profile, it has ideal pharmacokinetic properties for a hypoxia modifier, and has low cost ${ }^{18}$.

To evaluate the effect of atovaquone on other cell lines, the OCR was measured for $4.5 \mathrm{~h}$ after its addition. A significant decrease in the OCR of $78.7 \%$ to $90.0 \%$ compared with the DMSO control was observed after $2 \mathrm{~h}$ at $10 \mu \mathrm{M}$ in FaDu, HCT116 colorectal carcinoma, DLD-1 colorectal adenocarcinoma, H1299 lung carcinoma, A549 lung carcinoma, H460 large cell lung carcinoma, MCF7 breast ductal carcinoma and T24 bladder carcinoma cells, with a lesser but still significant decrease of $57.6 \%$ in PSN-1 pancreatic adenocarcinoma cells (Fig. 1c-e and Supplementary Fig. 1a). This was a dose-dependent effect, with a smaller, but still significant, decrease observed at $2 \mu \mathrm{M}$, and no significant decrease observed at $1 \mu \mathrm{M}$. There was no significant effect on the cell number relative to the DMSO control in FaDu, HCT116 and H1299 cells at concentrations between 10 and $100 \mu \mathrm{M}$, or in the other cell lines at $10 \mu \mathrm{M}$ during the course of the experiment (Supplementary Fig. 1b,c). Statistically significant decreases in cell survival of $25.9-56.2 \%$ were observed in $\mathrm{FaDu}$, HCT116 and H1299 cells after a longer incubation of $24 \mathrm{~h}$ with 20 or $30 \mu \mathrm{M}$ atovaquone, but cell survival was partially rescued following 4 days recovery in drug-free medium (Supplementary Fig. 1d). The widely used anti-cancer therapeutics, 5-fluorouracil, docetaxel and cytarabine caused a significant decrease in $\mathrm{FaDu}$ cell survival of $18.7-37.8 \%$ after $24 \mathrm{~h}$ incubation at either $2 \mu \mathrm{M}$ or 10 , but this did not correspond with a significant decrease in the OCR (Supplementary Fig. 2a,b). This demonstrates that a decrease in cell survival observed following protracted incubation with an anti-proliferative drug does not necessarily correlate with a decrease in the OCR. Thus atovaquone reduces the OCR in cancer cells from a wide range of tumour types.

ATO affects spheroid hypoxia and radiation response. Reduction of the OCR in 3D multicellular tumour spheroids can lead to a decrease in hypoxia ${ }^{6-8}$. FaDu, HCT116 and H1299 spheroids of $550-650 \mu \mathrm{m}$ in diameter were treated with 10,20 or $30 \mu \mathrm{M}$ atovaquone for $24 \mathrm{~h}$, and the hypoxic fraction was evaluated by 
a

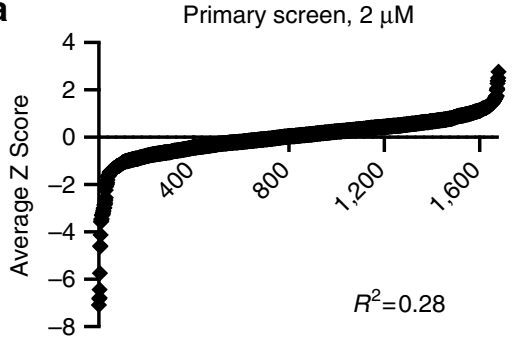

b

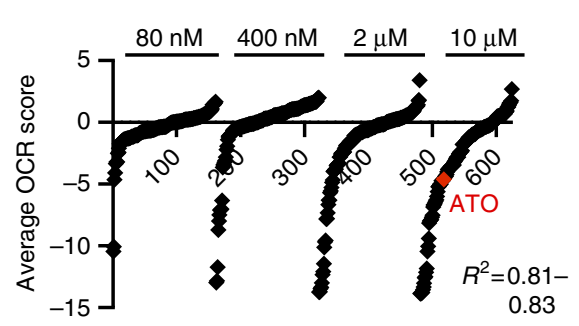

Primary screen, $10 \mu \mathrm{M}$

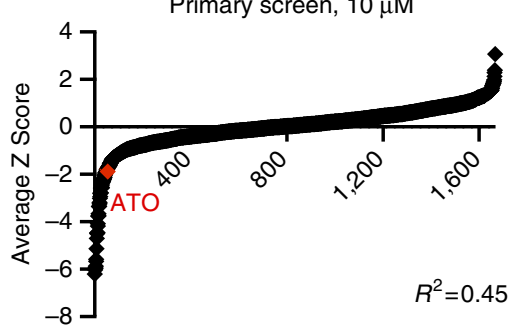

C

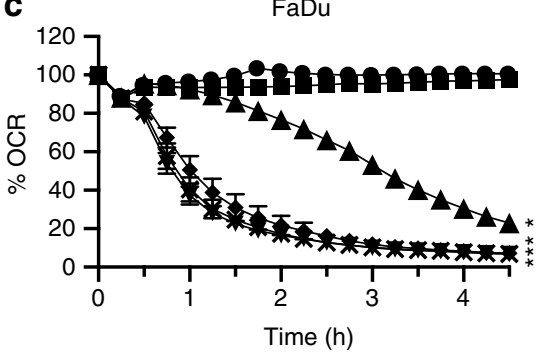

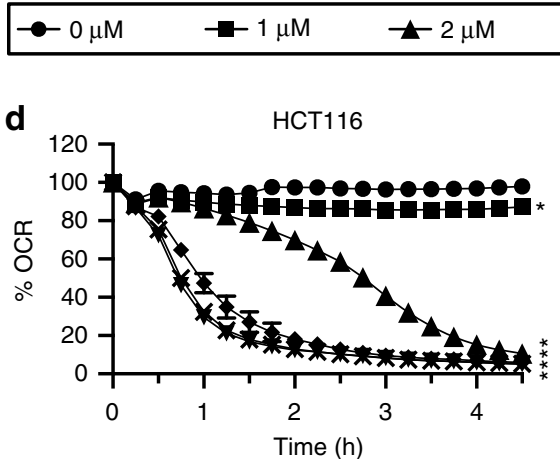

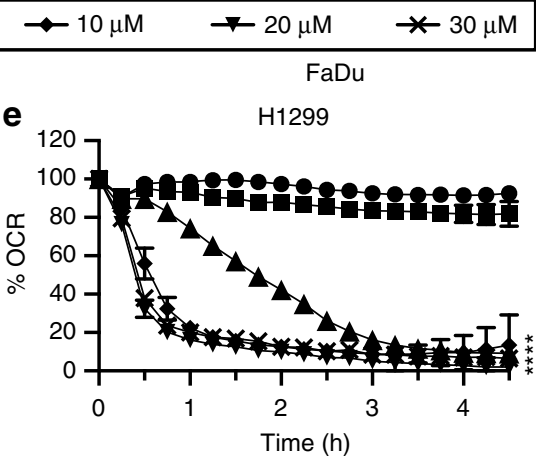

Figure 1 | A screen to discover compounds that decrease the OCR of FaDu cells. (a) For the primary screen, FaDu cells were grown in galactosecontaining medium and incubated with a library of 1697 FDA-approved compounds for $24 \mathrm{~h}$. The mitochondrial specific OCR was measured, and subsequent hoechst staining was used to correct for cell number. (b) For the secondary screen, FaDu cells were grown in glucose-containing medium, incubated with the compounds for $24 \mathrm{~h}$, and then the mitochondrial-specific OCR was measured and normalized to cell number. (c-e) The OCR of FaDu, HCT116 and H1299 cells were measured for $4.5 \mathrm{~h}$ after injection of atovaquone. The \% OCR post injection is shown relative to the DMSO control and normalized to the relative cell number obtained by hoechst staining at the end of the experiment. The data are representative of three independent experiments (mean \pm s.d.). One-way ANOVA were performed to assess statistical significance at $4.5 \mathrm{~h}$ after injection of atovaquone with Bonferroni post correction $\left({ }^{\star} P<0.0001\right)$. ATO, atovaquone.

\section{Table 1 | The highest ranked compounds that reduce the} OCR of FaDu cells at $10 \mu \mathrm{M}$.

\begin{tabular}{ll} 
Rank & Compound \\
\hline 1 & Pyrvinium \\
2 & Berberine \\
3 & Niclosamide \\
4 & Acriflavinium \\
5 & Sorafenib \\
6 & Emetine \\
7 & Plicamycin \\
8 & Suloctidil \\
9 & Pentamidine \\
10 & Amsacrine \\
11 & Phenformin \\
12 & Irinotecan \\
13 & Itraconazole \\
14 & Mitomycin \\
15 & Atovaquone \\
16 & Hydroxyprogesterone \\
17 & Cyclosporine \\
18 & Fenofibrate \\
\hline
\end{tabular}

EF5 staining. There was complete loss of hypoxia in FaDu spheroids at $30 \mu \mathrm{M}$, and in HCT116 and H1299 spheroids at $20 \mu \mathrm{M}$ atovaquone (Fig. 2a,b). It is possible that a decrease in EF5 staining could be caused by an increase in cell death ${ }^{7}$. To exclude this possibility, the spheroids were washed and allowed to recover in drug-free medium for $24 \mathrm{~h}$, resulting in the return of hypoxia (Fig. 2a,b). None of the treatments caused a significant decrease in the diameter of $\mathrm{FaDu}$, HCT116 or H1299 spheroids (Fig. 2c). Taken together, these results indicate that the observed decrease in the OCR induced by atovaquone corresponds to a decrease in hypoxia in an avascular 3D tumour environment.

To determine whether the alleviation of spheroid hypoxia translated to an improvement in radiation response, $\mathrm{FaDu}$ spheroids were treated for $24 \mathrm{~h}$ with $30 \mu \mathrm{M}$ atovaquone, irradiated at $10 \mathrm{~Gy}$, and then allowed to recover in fresh medium. For unirradiated spheroids, atovaquone treatment did not cause a significant difference in spheroid growth after 20 days (Fig. 2d). Irradiation at $10 \mathrm{~Gy}$ caused dissociation of both the DMSO and atovaquone-treated spheroids, but $90.91 \%$ of the DMSO-treated spheroids reformed by 60 days after irradiation, compared with only $37.93 \%$ of atovaquone treated spheroids 
a

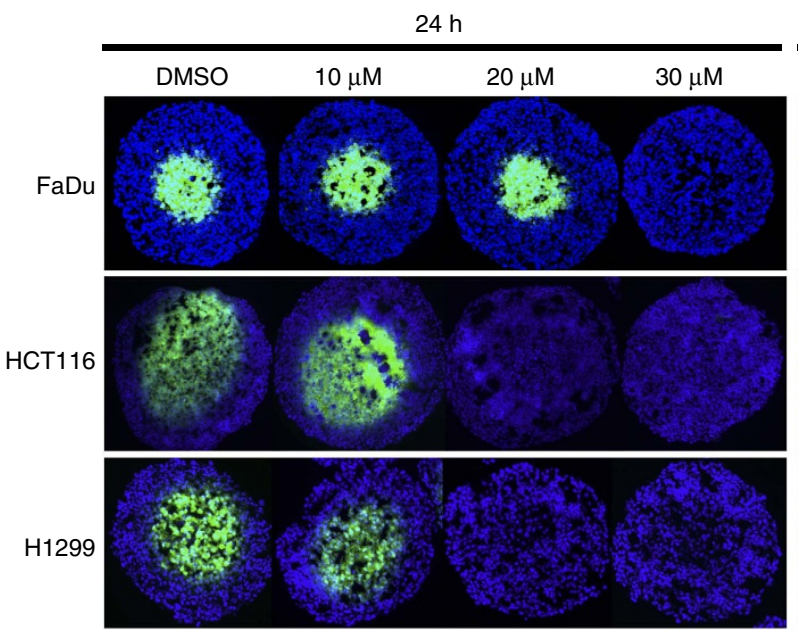

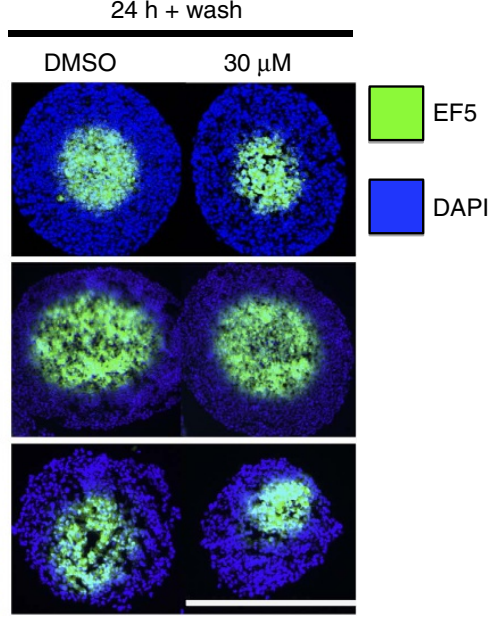

b
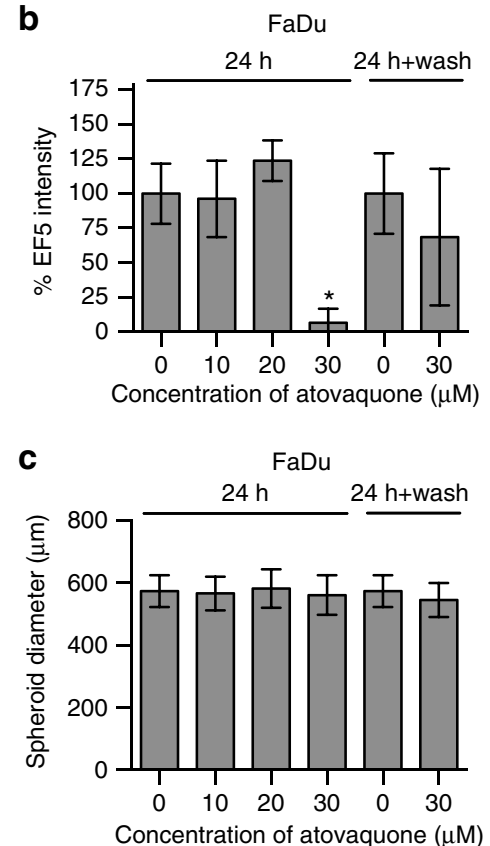

HCT116

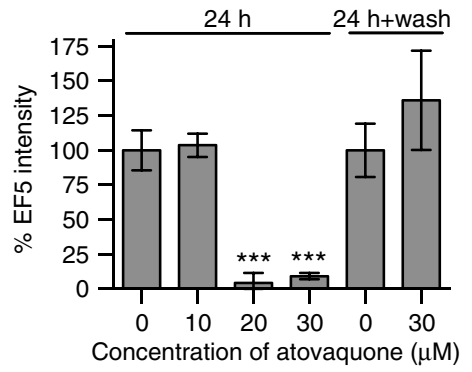

HCT116

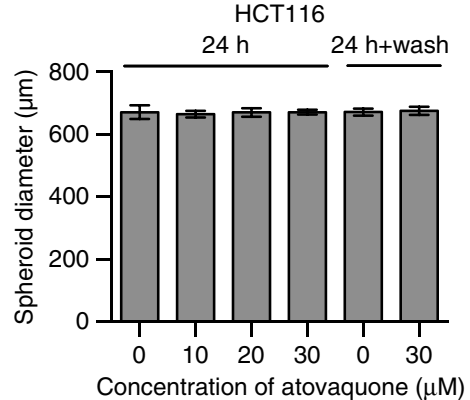

H1299

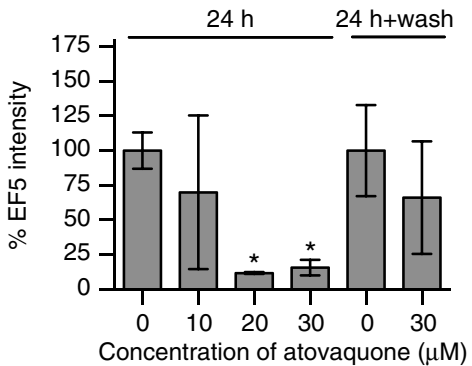

H1299

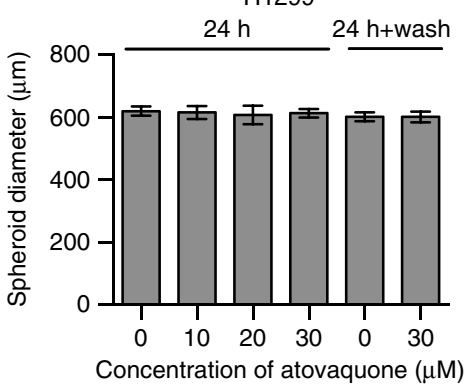

e
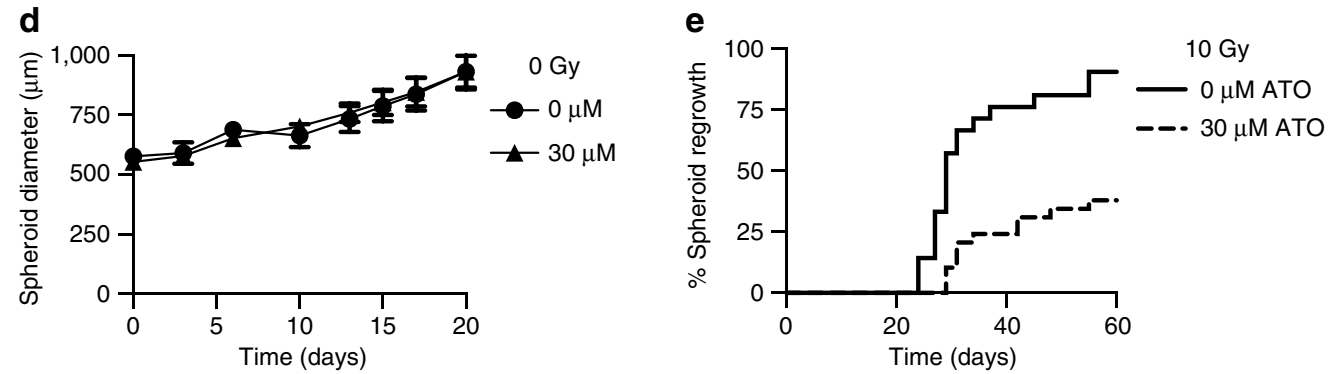

Figure 2 | Atovaquone alleviates spheroid hypoxia and improves radiation response. (a,b) FaDu, HCT116 and H1299 spheroids were treated with DMSO or atovaquone either for $24 \mathrm{~h}$, or for $24 \mathrm{~h}$ followed by recovery for $24 \mathrm{~h}$ in drug-free medium, as indicated. Hypoxia was assessed by staining central spheroid sections for EF5 (green), with DAPI as a nuclear counterstain (blue). Scale bar, $600 \mu \mathrm{m}$. \% Mean EF5 fluorescence intensity is presented relative to the DMSO controls. (c) Average spheroid diameter. Hypoxia was assessed in at least five spheroids per treatment for each experiment ( $n=3$ ). One-way ANOVA was performed to assess statistical significance with Bonferroni post correction ( $P>0.05$, ${ }^{\star \star \star} P<0.001$ ). (d,e) FaDu spheroids were treated with DMSO or $30 \mu \mathrm{M}$ atovaquone for $24 \mathrm{~h}$, and then received either $0 \mathrm{~Gy}$ (d) or $10 \mathrm{~Gy}(\mathbf{e})$. After treatment, the spheroids were allowed to recover in drug-free medium. Time post treatment is shown (days). One replicate of the experiment was conducted with at least 22 spheroids in each treatment group. All values are presented as mean \pm s.d.

(Fig. 2e). This decrease in irradiated spheroid regrowth following atovaquone treatment is likely due to the observed reduction in spheroid hypoxia.
ATO inhibits mitochondrial complex III. As a ubiquinone analogue, the primary clinical mechanism of action of atovaquone is inhibition of Plasmodium and Pneumocystis complex III 
(refs 19-21). To investigate whether atovaquone also inhibits complex III in human cancer cells, the activity of the ETC complexes I-IV was assessed in FaDu cells permeabilized with digitonin. Digitonin $(0.005 \%)$ was sufficient to permeabilize the cells, allowing respiration of succinate, a substrate that does not cross intact cell membranes (Supplementary Fig. 2c). Complex I-dependent respiration was significantly inhibited by $30 \mu \mathrm{M}$ atovaquone, the complex III inhibitor, myxothiazol and the complex I inhibitor, rotenone (Fig. 3a). Complex II-dependent respiration was significantly inhibited by $30 \mu \mathrm{M}$ atovaquone, myxothiazol and the complex II inhibitor, malonate. Complex III-dependent respiration was significantly inhibited by $30 \mu \mathrm{M}$ atovaquone, myxothiazol and the complex III inhibitor, antimycin A. Complex IV-dependent respiration was significantly inhibited by the complex IV inhibitor, sodium azide, but not by $30 \mu \mathrm{M}$ atovaquone, and was less significantly inhibited by myxothiazol. Taken together, these results suggest that the primary mechanism of action of atovaquone in $\mathrm{FaDu}$ cells is inhibition of complex III, which then causes a decrease in the activity of the upstream ETC complexes.

To study complex III in isolation from the other ETC complexes, the reduction of excess cytochrome $c$ was measured in mitochondria isolated from $\mathrm{FaDu}$ cells, a reaction catalysed by complex III. There was a significant decrease in complex II/III activity by the complex II inhibitor, malonate, and by the complex III inhibitors, antimycin, myxothiazol and $30 \mu \mathrm{M}$ atovaquone (Fig. 3b). Due to the dependence of this assay on complex II activity, a complex II-specific assay was performed in parallel using mitochondria isolated from $\mathrm{FaDu}$ cells. There was a significant decrease in complex II activity by the complex II inhibitor, malonate (Fig. 3c). However, there was no inhibition of complex II activity by the complex III inhibitors, antimycin,

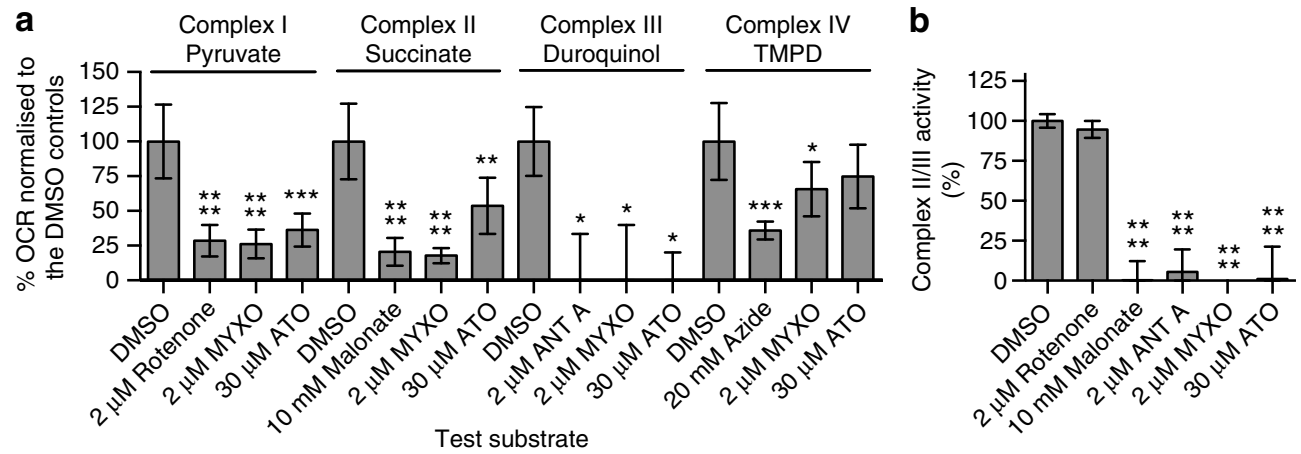

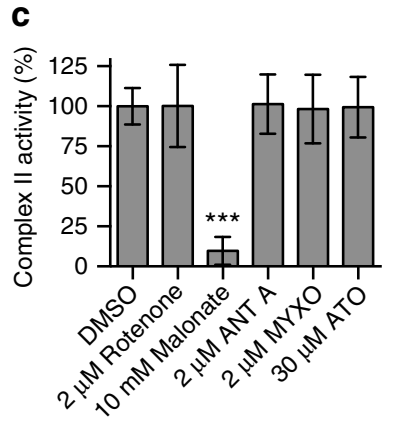

Test substrate
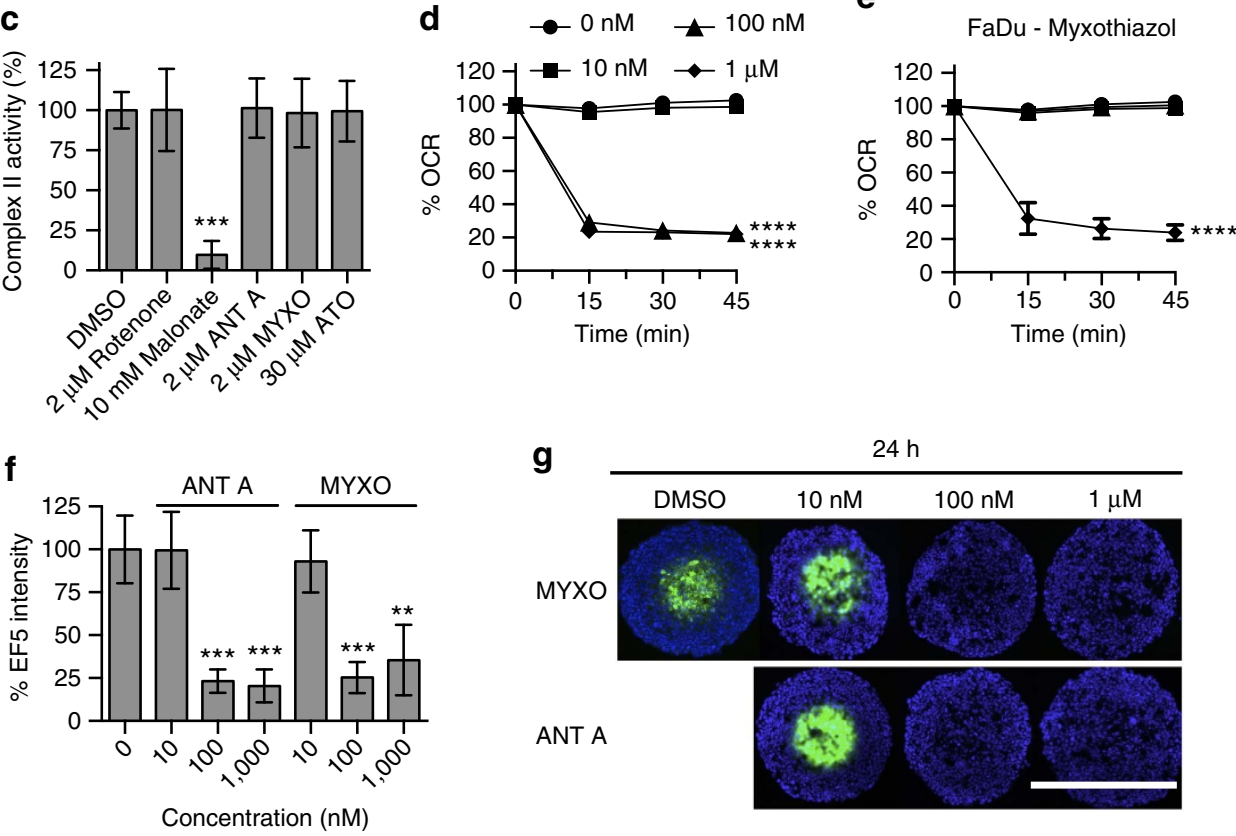

Figure 3 | Atovaquone inhibits complex III activity. (a) The effect of atovaquone on complex I-, II-, III- or IV-dependent respiration in FaDu cells permeabilized with $0.005 \%$ digitonin. The \% OCR was measured immediately after permeabilization. (b) Complex II/III activity was measured in mitochondria isolated from FaDu cells 15 min after compound addition. (c) Complex II activity was measured in mitochondria isolated from FaDu cells 15 min after compound addition. (d,e) The OCR of FaDu cells was measured for 45 min after injection of antimycin A (d) or myxothiazol (e). The \% OCR post injection is shown relative to the DMSO control and normalized to the relative cell number obtained by hoechst staining at the end of the experiment.

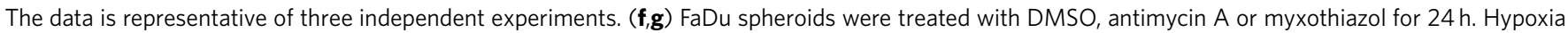
was assessed by staining central spheroid sections for EF5 (green), with DAPI as a nuclear counterstain (blue). Scale bar, $600 \mu$ m. \% Mean EF5 fluorescence intensity is presented relative to the DMSO controls. Hypoxia was assessed in at least five spheroids per treatment for each experiment $(n=3)$. One-way ANOVA with Bonferroni was performed for all of the experiments $\left(n=3\right.$, mean $\left.\pm \mathrm{s} . \mathrm{d}_{.},{ }^{\star \star \star \star} P<0.0001,{ }^{\star \star \star} P<0.001,{ }^{\star \star} P<0.01,{ }^{\star} P<0.05\right)$. ANT A, antimycin A; ATO, atovaquone; MYXO, myxothiazol. 
myxothiazol or $30 \mu \mathrm{M}$ atovaquone. These findings were also observed in mitochondria isolated from bovine heart (Supplementary Fig. 2d,e). To demonstrate that complex III inhibition is sufficient to reduce the OCR, FaDu cells were incubated with antimycin A or myxothiazol and the OCR was measured for $45 \mathrm{~min}$. A rapid and significant decrease in the OCR was observed in a dose-dependent manner for both inhibitors, with no decrease at $10 \mathrm{nM}$, but a significant decrease at $1 \mu \mathrm{M}$ (Fig. 3d,e). Furthermore, incubation of $\mathrm{FaDu}$ spheroids for $24 \mathrm{~h}$ with $1 \mu \mathrm{M}$ antimycin or myxothiazol eliminated spheroid hypoxia without affecting spheroid size, whereas no reduction in hypoxia was observed at $10 \mathrm{nM}$ (Fig. 3f,g and Supplementary Fig. 2f). These findings indicate that atovaquone specifically inhibits complex III in FaDu cells, and that complex III inhibition is sufficient to reduce both the OCR and spheroid hypoxia.

ATO inhibits pyrimidine synthesis. Dihydroorotate dehydrogenase (DHODH) is an enzyme of the de novo pyrimidine synthesis pathway situated on the inner mitochondrial membrane (Fig. 4a). In biochemical assays, atovaquone inhibits not only complex III, but also DHODH ${ }^{22}$. To investigate whether a known DHODH inhibitor could reduce the OCR, FaDu cells were incubated for $2 \mathrm{~h}$ with leflunomide ${ }^{23,24}$, which caused a dose-dependent decrease in the OCR (Fig. 4b). However, inhibition of the pyrimidine synthesis pathway downstream of DHODH by the orotidylic acid decarboxylase inhibitor, 6-azauridine (6-Aza), or by the ribonucleotide reductase inhibitor, hydroxyurea ${ }^{25,26}$ did not cause a decrease in the OCR of FaDu cells after $24 \mathrm{~h}$ incubation (Fig. 4c).
The levels of nucleoside triphosphates were measured by high-performance liquid chromatography (HPLC) in FaDu cells treated for $24 \mathrm{~h}$ with atovaquone or 6 -Aza. In the cells treated with atovaquone, there was a significant decrease in UTP levels, a non-significant decrease in CTP levels, and no significant changes in GTP or ATP levels (Fig. 4d and Supplementary Fig. 2g). Therefore atovaquone appears to specifically affect pyrimidine synthesis. In the cells treated with 6-Aza, there were significant decreases in UTP and CTP levels, a non-significant decrease in GTP levels, and a non-significant increase in ATP levels, indicating that 6-Aza primarily affects pyrimidine synthesis under these conditions. Furthermore, the observed decrease in the OCR induced by incubation with atovaquone for $2 \mathrm{~h}$ was not rescued by addition of the pyrimidine precursor, uridine, in $\mathrm{FaDu}$ cells (Fig. 4e). These results suggest that inhibition of DHODH by atovaquone causes a decrease in both the OCR and pyrimidine synthesis, but that the decrease in pyrimidine synthesis is unlikely to cause the decrease in the OCR.

ATO doesn't affect HIF-1 $\alpha$ or intrinsic radiosensitivity. HIF-1 is a heterodimeric transcription factor that promotes angiogenesis and increased proliferation ${ }^{1}$. The HIF-1 $\alpha$ subunit is rapidly degraded under normoxia, but is stabilized under hypoxia. To assess the effect of atovaquone on HIF- $1 \alpha$ expression in vitro, $\mathrm{FaDu}, \mathrm{HCT} 116$ and $\mathrm{H} 1299$ cells were incubated with atovaquone for 2 or $24 \mathrm{~h}$ in either normoxic $\left(20 \% \mathrm{O}_{2}\right)$ or hypoxic $\left(0.5 \% \mathrm{O}_{2}\right)$ conditions. HIF- $1 \alpha$ was not stabilized by atovaquone under normoxic conditions and no significant differences in HIF-1 $\alpha$
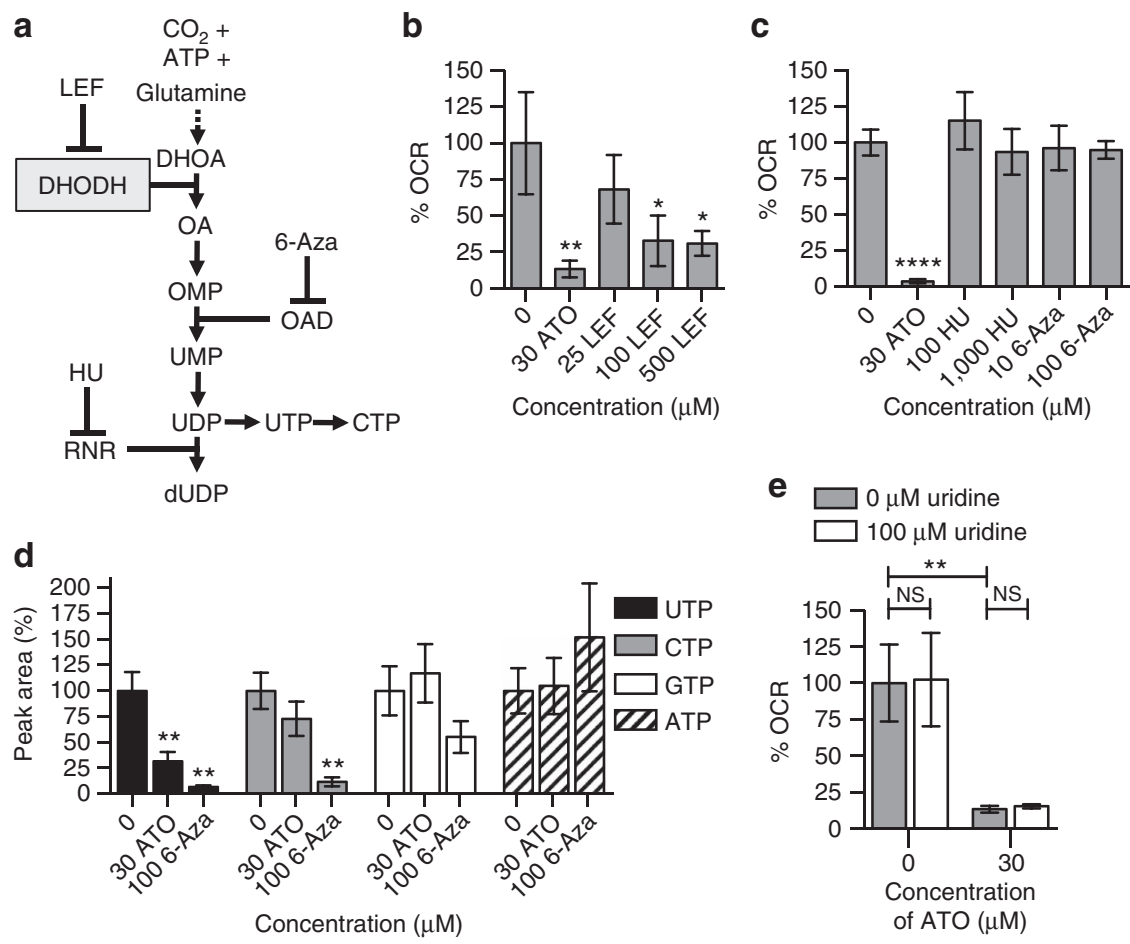

Figure 4 | Atovaquone inhibits pyrimidine synthesis. (a) The pyrimidine synthesis pathway. 6-Aza, 6-azauridine; DHODH, dihydroorotate dehydrogenase; HU, hydroxyurea; LEF, leflunomide; OAD, orotidylic acid decarboxylase; RNR, ribonucleotide reductase. (b) The \% OCR following $2 \mathrm{~h}$ incubation of FaDu cells with LEF or $30 \mu \mathrm{M}$ ATO. (c) The \% OCR following $24 \mathrm{~h}$ incubation of FaDu cells with HU, 6-Aza or $30 \mu \mathrm{M}$ ATO. (d) NTP peak area levels relative to the DMSO control and normalized to cell number after $24 \mathrm{~h}$ incubation with $30 \mu \mathrm{M}$ ATO or $100 \mu \mathrm{M} 6$-Aza. (e) The \% OCR following $2 \mathrm{~h}$ incubation of FaDu cells with either $100 \mu \mathrm{M}$ uridine, $30 \mu \mathrm{M}$ ATO or both $100 \mu \mathrm{M}$ uridine and $30 \mu \mathrm{M} \mathrm{ATO} \%$ OCR is presented relative to the DMSO control and corrected for cell number $(n \geq 3)$. One-way ANOVA with Bonferroni was performed for all of the experiments apart from e, for which an unpaired two-tailed T-test was performed $\left({ }^{\star \star \star \star} P<0.0001,{ }^{\star \star \star} P<0.001,{ }^{\star \star} P<0.01,{ }^{\star} P<0.05\right)$. All values are presented as mean $\pm \mathrm{s}$.d. except for the HPLC data, which are presented as mean \pm s.e.m. ATO, atovaquone. 
protein levels were observed under hypoxic conditions (Supplementary Fig. 3a,b).

Radiation sensitivity can be affected not only by tumour hypoxia, but also by alteration of the intrinsic radiosensitivity of the cancer cells $s^{1,3,4}$. Therefore colony formation assays were conducted to investigate whether atovaquone alters intrinsic radiation sensitivity in monolayer FaDu, HCT116 or H1299 cells. Assessment of colony formation revealed that atovaquone did not alter radiosensitivity under either normoxia $\left(20 \% \mathrm{O}_{2}\right)$ or severe hypoxia $\left(<0.1 \% \mathrm{O}_{2}\right)$ (Supplementary Fig. $3 \mathrm{c}$ and Supplementary Table 2). This indicates that, as expected, atovaquone is not an intrinsic radiation sensitizer.

ATO affects tumour hypoxia and radiation response. Given that atovaquone completely alleviated spheroid hypoxia at pharmacologically relevant doses, mice bearing $\mathrm{FaDu}$ and HCT116 xenografts were treated with atovaquone for 7 days to investigate the effect on tumour hypoxia. The tumours were harvested on day 7, and EF5 staining confirmed that tumour hypoxia was virtually abolished in both the FaDu and HCT116 xenografts (Fig. 5a,b). As expected, the tumour volume was not significantly altered during the course of the experiment (Fig. 5c). The mean concentration of atovaquone in the blood plasma after 5 days treatment was $68.6 \mu \mathrm{M}$, and mean concentration in the HCT116 tumours was $36.0 \mu \mathrm{M}$ (Fig. 5d).

Given the dramatic decrease in tumour hypoxia observed following atovaquone treatment, radiation response was assessed in mice bearing $\mathrm{FaDu}$ xenografts. The mice were treated with atovaquone for 7 days prior to irradiation with a single dose of $6 \mathrm{~Gy}$, and then atovaquone treatment was continued for a further 3 days. In the unirradiated mice, the average time taken for the tumours to reach $500 \mathrm{~mm}^{3}$ was 14.2 days for mice treated with DMSO, and 12.3 days for mice treated with atovaquone, a non-significant difference, indicating that atovaquone alone does not alter tumour volume (Fig. 5e,f). In the irradiated mice, the average time taken for the tumours to reach $500 \mathrm{~mm}^{3}$ was 15.3 days for mice treated with DMSO, but 28.5 days for mice treated with atovaquone, a significant synergistic growth delay of 13.2 days between the two irradiated groups $(P<0.0001)$. One tumour, excluded from analysis, treated with both atovaquone and radiation had an excellent response, reaching $500 \mathrm{~mm}^{3} 42$ days after the initiation of drug treatment. The observed tumour growth delay is likely due to the reduction in tumour hypoxia observed with atovaquone treatment.

Biguanides affect spheroid hypoxia and radiation response. Previous publications have demonstrated that the anti-diabetic biguanide, metformin, reduces the OCR and tumour hypoxia ${ }^{11,12}$. To evaluate the relative efficacy of atovaquone, metformin and its precursor, phenformin, the OCR was measured in FaDu, HCT116 and H1299 cells following $24 \mathrm{~h}$ treatment. A significant decrease in the OCR of over $90.0 \%$ was observed in all three cells lines treated with $30 \mu \mathrm{M}$ atovaquone (Fig. 6a). A significant decrease of $70.7 \%$ in FaDu cells and 59.0\% in HCT116 cells was observed at $2 \mathrm{mM}$ metformin. However, metformin was less potent in H1299 cells, causing a decrease of $33.1 \%$ at $2 \mathrm{mM}$, which was not statistically significant. A significant decrease in the OCR of $52.2 \%$ in FaDu cells and $43.3 \%$ in HCT116 cells was observed at $10 \mu \mathrm{M}$ phenformin. Phenformin was also less potent in H1299 cells, causing a non-significant decrease of $23.4 \%$. There were no significant changes in the OCR at 200 or $20 \mu \mathrm{M}$ metformin, or at 2 or $0.4 \mu \mathrm{M}$ phenformin in any of the cell lines. Metformin and phenformin did not affect cell survival at any concentration tested (Supplementary Fig. 4a). To investigate the kinetics of this effect in FaDu cells, the OCR was measured for $15 \mathrm{~h}$ after injection of atovaquone, metformin or phenformin. The OCR was reduced by $87.3 \%$ by atovaquone within $2 \mathrm{~h}$ (Supplementary Fig. $4 \mathrm{~b}$ ). However, a slower decrease in the OCR was observed following treatment with $2 \mathrm{mM}$ metformin or $10 \mu \mathrm{M}$ phenformin, with a reduction of $<10 \%$ within $2 \mathrm{~h}$ and a reduction of $55.9 \%$ and $36.0 \%$, respectively, after $10 \mathrm{~h}$.

To assess whether the observed decrease in the OCR translated to a decrease in spheroid hypoxia, FaDu, HCT116 and H1299 spheroids were treated with atovaquone, metformin or phenformin for $24 \mathrm{~h}$, and the hypoxic fraction was evaluated by EF5 staining. There was near-complete loss of hypoxia in FaDu and HCT116 spheroids at $30 \mu \mathrm{M}$ atovaquone, $2 \mathrm{mM}$ metformin and $10 \mu \mathrm{M}$ phenformin (Fig. 6b,c, Supplementary Fig. 4c). In contrast, there were non-significant decreases of $41.8 \%$ and $9.8 \%$ in $\mathrm{H} 1299$ spheroids following treatment with $2 \mathrm{mM}$ metformin and $10 \mu \mathrm{M}$ phenformin, respectively. No significant decreases in hypoxia were observed at $20 \mu \mathrm{M}$ or $200 \mu \mathrm{M}$ metformin, or at $0.4 \mu \mathrm{M}$ or $2 \mu \mathrm{M}$ phenformin in $\mathrm{FaDu}$ spheroids. No treatments caused a significant change in spheroid diameter (Supplementary Fig. 4d). These results are in accordance with the OCR results shown in Fig. 6a, suggesting that a significant reduction in the OCR by metformin and phenformin is required to reduce spheroid hypoxia.

Nimorazole is a 2-nitroimidazole that acts as an oxygen mimetic, which has previously been shown to improve loco-regional control when combined with radiotherapy in head and neck cancer ${ }^{27,28}$. To compare the ability of nimorazole, atovaquone, metformin, phenformin and antimycin $\mathrm{A}$ to improve radiation response, $\mathrm{FaDu}$ spheroids were treated for $24 \mathrm{~h}$ with these drugs, irradiated at $10 \mathrm{~Gy}$, and then allowed to recover in fresh medium. Fifty days after irradiation, $90.5 \%$ of the DMSO-treated spheroids were reformed (Fig. 6d). In comparison, the percentage reformation of spheroids for the highest concentration of each treatment was $53.7 \%$ for $10 \mathrm{mM}$ nimorazole, $48.6 \%$ for atovaquone, $58.3 \%$ for $2 \mathrm{mM}$ metformin, $53.2 \%$ for $10 \mu \mathrm{M}$ phenformin, and $50.5 \%$ for $1 \mu \mathrm{M}$ antimycin A (Fig. 6d,e). A less-dramatic decrease in spheroid regrowth was observed at the lower concentrations of the drugs, with a percentage spheroid reformation of $72.1 \%$ for $1 \mathrm{mM}$ nimorazole, $75.6 \%$ for $20 \mu \mathrm{M}$ metformin, $75.0 \%$ for $0.4 \mu \mathrm{M}$ phenformin and $60.4 \%$ for $0.1 \mu \mathrm{M}$ antimycin A. For unirradiated spheroids, no drug treatment caused a significant decrease in spheroid growth compared with the DMSO control after 35 days (Supplementary Fig. 5a,b). These results demonstrate that the greatest improvement in radiation response is observed at concentrations of the drugs that eliminate spheroid hypoxia, and that this is sufficient to improve radiation response in a manner comparable to $10 \mathrm{mM}$ nimorazole.

Having demonstrated that metformin and phenformin reduce hypoxia in spheroids, their effect on hypoxia was next examined in vivo. Mice bearing FaDu xenografts were treated with DMSO for 7 days, $50 \mathrm{mg} \mathrm{kg}^{-1}$ atovaquone for 7 days, a single dose of $250 \mathrm{mg} \mathrm{kg}^{-1}$ metformin, $250 \mathrm{mg} \mathrm{kg}^{-1}$ per day metformin for 7 days or $100 \mathrm{mg} \mathrm{kg}^{-1}$ per day phenformin for 7 days. The tumours were harvested $2.5 \mathrm{~h}$ after the final dose of drug, and EF5 staining was performed. Atovaquone caused a significant $73.7 \%$ reduction in hypoxia, and 7 days metformin treatment caused a $43.1 \%$ decrease in hypoxia, although this was not statistically significant. There were no significant changes in hypoxia following a single dose of metformin or 7 days phenformin (Fig. 6f and Supplementary Fig. 5c). After a single dose of metformin the mean plasma concentration was $109 \mu \mathrm{M}$, and mean tumour concentration was $151 \mu \mathrm{M}$ (Supplementary Fig. 5d).

\section{Discussion}

Our screen, designed to find compounds that decrease tumour hypoxia by inhibiting oxygen consumption, identified 
a

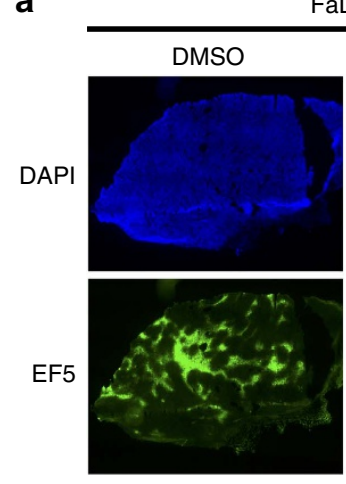

$\mathrm{FaDu}$

HCT116
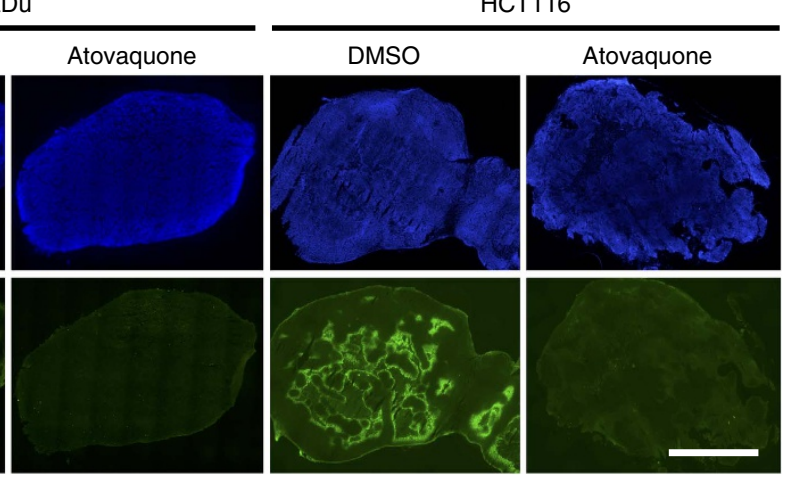

b

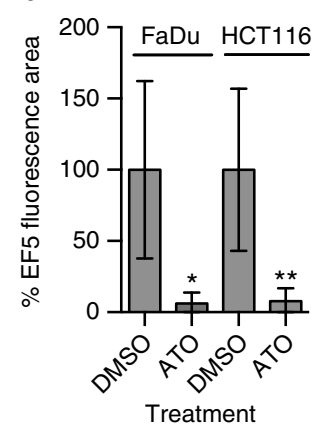

C

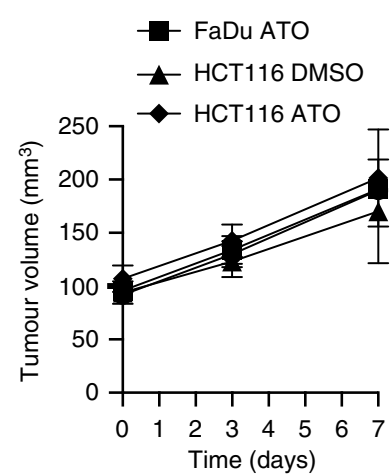

d

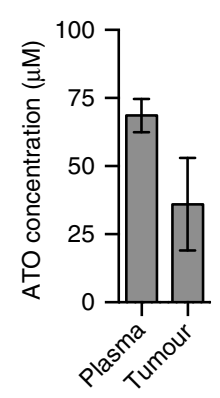

。
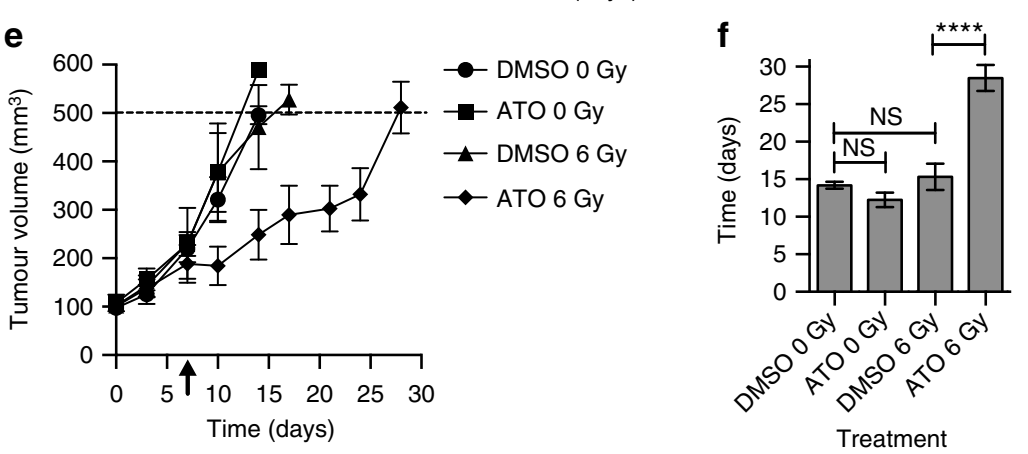

Figure 5 | ATO reduces tumour hypoxia and improves tumour radiation response. Mice bearing FaDu and $\mathrm{HCT116}$ xenograft tumours were treated with atovaquone for 7 days, and were injected with EF5 on day 7. (a) Immunostaining for EF5 with DAPI as a nuclear counterstain. Scale bar, 2 mm. (b) \% EF5

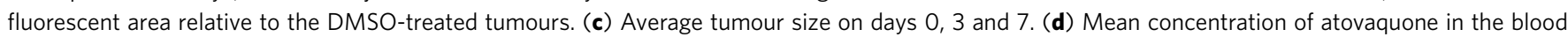
plasma after 5 days treatment, and mean concentration in the excised HCT116 tumours. (e) Mice bearing FaDu xenograft tumours were treated with atovaquone for 10 days, with 6 Gy radiation on day 7 (indicated by arrow). Average tumour volume is shown (mm ${ }^{3}$ ). One tumour from a mouse treated with atovaquone and radiation took 42 days to reach $500 \mathrm{~mm}^{3}$ and was excluded from analysis. (f) Average time taken for tumours to reach the maximum volume of $500 \mathrm{~mm}^{3}$ (days). At least five mice were used for each treatment group. One-way ANOVA were performed to assess statistical significance with Bonferroni post correction ( ${ }^{\star \star \star \star} P<0.0001,{ }^{\star \star} P<0.01,{ }^{\star} P<0.05$ ). All values are presented as mean $\pm \mathrm{s}$.d. except for the HPLC data, which are presented as mean \pm s.e.m. ATO, atovaquone.

atovaquone. Atovaquone caused a rapid decrease in oxygen consumption in a wide range of cancer cell lines. It virtually abolished both spheroid hypoxia and tumour hypoxia, and augmented the effects of radiotherapy. Its mechanism of action is likely the inhibition of the mitochondrial cytochrome $b c_{1}$ complex (complex III).

The efficacy of radiotherapy can be improved by the reoxygenation of radioresistant hypoxic tumour cells $s^{1,3,4}$. This study reveals that atovaquone inhibits oxygen consumption, spheroid hypoxia and tumour hypoxia, but has no effect on intrinsic radiosensitivity in vitro under normoxic or hypoxic conditions. However, atovaquone improved radiation sensitivity in both spheroids and xenograft tumours. Therefore we propose that the radiation sensitization following atovaquone treatment could be due to a decrease in hypoxia rather than an increase in intrinsic radiosensitivity. It is not expected that atovaquone would exacerbate radiation-induced side effects in a clinical setting because irradiated normal tissues are not generally hypoxic. The improvement in radiation response in spheroids was comparable to $10 \mathrm{mM}$ nimorazole, although the percentage reformation of spheroids was $72.1 \%$ for $1 \mathrm{mM}$ nimorazole, compared with $48.6 \%$ for atovaquone, and the concentration of nimorazole in the blood plasma of patients is in the sub-millimolar range ${ }^{29-31}$. This indicates that the improvement of radiation response in spheroids is superior for atovaquone compared with nimorazole at pharmacological concentrations.

Atovaquone is FDA approved as a single agent to treat pneumocystis pneumonia, which is caused by Pneumocystis 
a

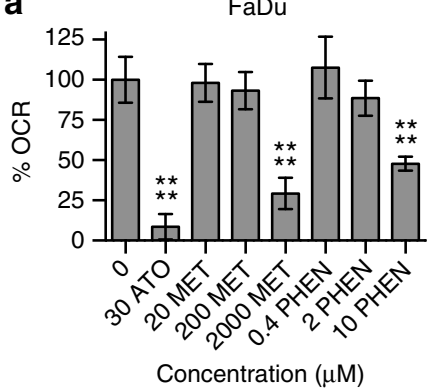

HCT116

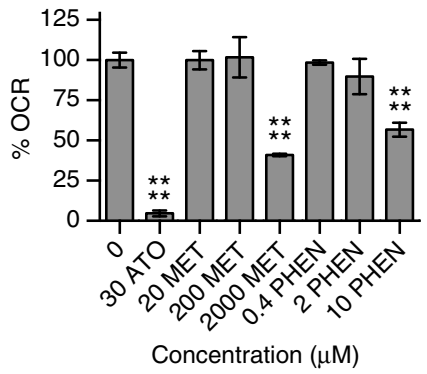

H1299

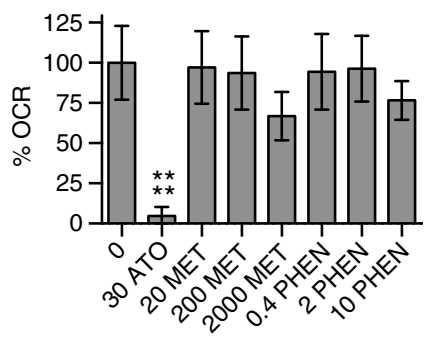

Concentration $(\mu \mathrm{M})$

b

MET PHEN

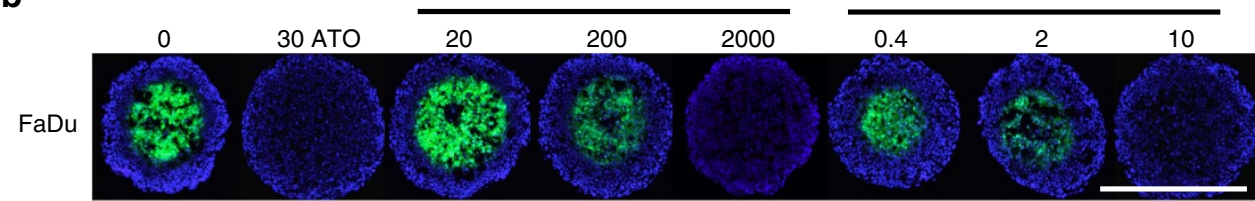

C
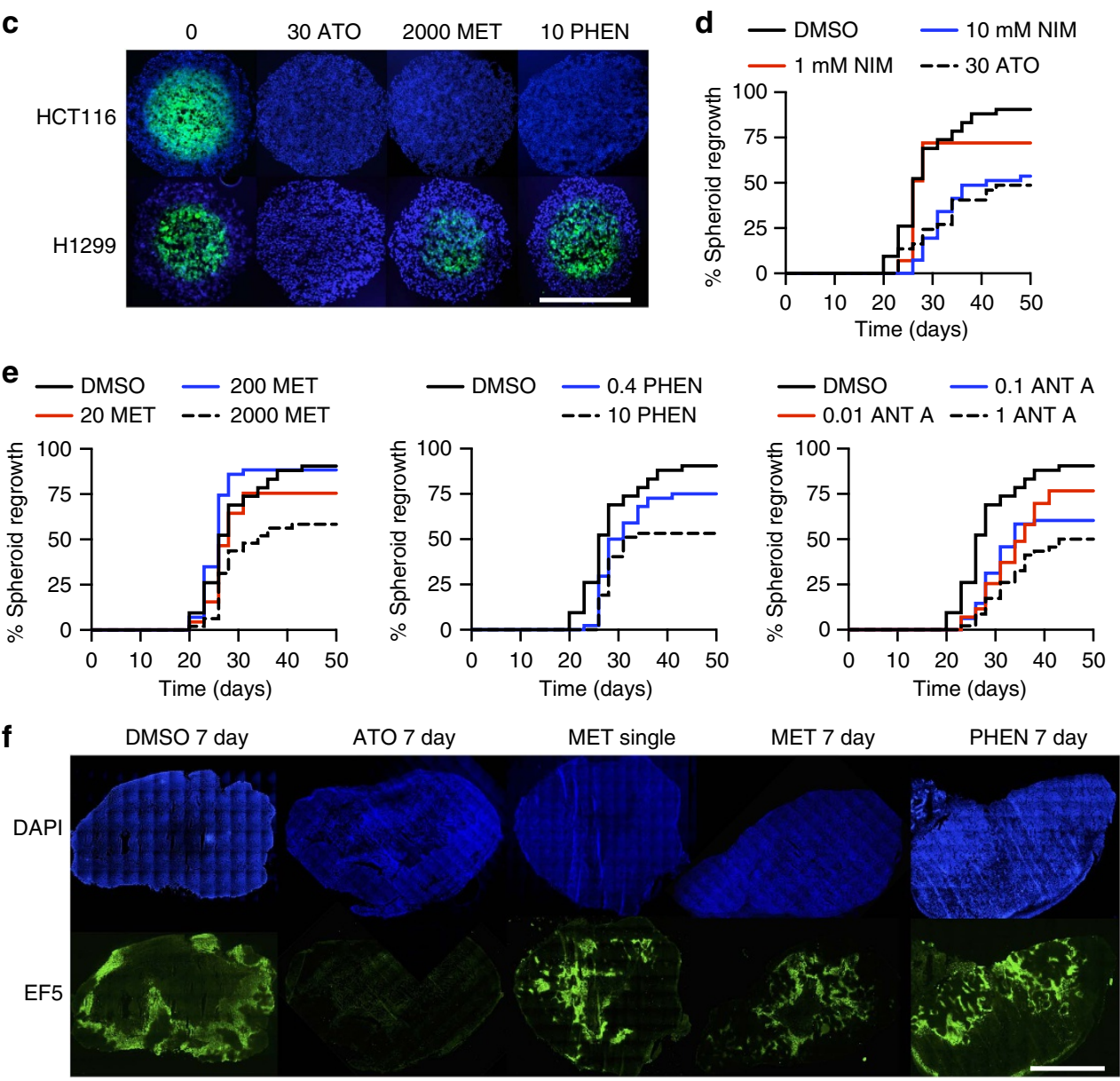

Figure 6 | Biguanides alleviate spheroid hypoxia and improve radiation response. (a) The OCR of FaDu, HCT116 and H1299 cells were measured after $24 \mathrm{~h}$ incubation with atovaquone, metformin or phenformin at the indicated concentrations. The \% OCR is shown relative to the DMSO control and normalized to the relative cell number obtained by hoechst staining at the end of the experiment. The data are an average of three independent experiments. One-way ANOVA were performed to assess statistical significance with Bonferroni post correction (mean \pm s.d., $\left.{ }^{\star \star \star \star P}<<0.0001\right) .(\mathbf{b}, \mathbf{c})$ FaDu $(\mathbf{b}), \mathrm{HCT116}$ and $\mathrm{H} 1299$ (c) spheroids were treated with DMSO, atovaquone, metformin or phenformin for $24 \mathrm{~h}$ at the indicated concentrations ( $\mu$ M). Hypoxia was assessed by staining central spheroid sections for EF5 (green), with DAPI as a nuclear counterstain (blue). Scale bar, $600 \mu$ m. Hypoxia was assessed in at least five spheroids per treatment for each experiment $(n=3)$, and the data are representative of three independent experiments. (d,e) FaDu spheroids were treated with DMSO, atovaquone, nimorazole, antimycin A, metformin or phenformin for $24 \mathrm{~h}$ at the indicated concentrations $(\mu \mathrm{M})$. After $24 \mathrm{~h}$ the spheroids received $10 \mathrm{~Gy}$, and were then allowed to recover in drug-free medium. Time post treatment is shown (days). One replicate of the experiment was conducted with at least 37 spheroids in each treatment group. (f) Mice bearing FaDu xenograft tumours were treated with $50 \mathrm{mg} \mathrm{kg}^{-1}$ per day atovaquone for 7 days, $250 \mathrm{mg} \mathrm{kg}^{-1}$ metformin single dose, $250 \mathrm{mg} \mathrm{kg}^{-1}$ per day metformin for 7 days or $100 \mathrm{mg} \mathrm{kg}^{-1}$ per day phenformin for 7 days. EF5 was injected 30 min after the final dose, and then the mice were killed after a further $2 \mathrm{~h}$. Immunostaining for EF5 with DAPI as a nuclear counterstain is shown. Scale bar, $2 \mathrm{~mm}$. Five mice were used for each treatment group. ANT A, antimycin A; ATO, atovaquone; MET, metformin; NIM, nimorazole; PHEN, phenformin. 
jirovecii $^{18}$. It is also used, primarily in combination with proguanil, as chemoprophylaxis against malaria, or to treat malaria, which is caused by Plasmodium falciparum. Atovaquone has a good safety profile with only mild side effects, is well absorbed, can accumulate in tissues, is eliminated by the liver after $50-84 \mathrm{~h}$, and is not metabolized in vivo to a significant extent ${ }^{18,32-34}$. It is an analogue of the electron carrier, ubiquinone, which is reduced to ubiquinol by $\mathrm{DHODH}$, complex I and complex II, and is then oxidized back to ubiquinone by complex III (ref. 18). In parasites, the primary mechanism of action of atovaquone is inhibition of complex III (refs 20,21). In biochemical assays, atovaquone inhibits $P$. falciparum complex III with an $\mathrm{IC}_{50}$ of $3 \mathrm{nM}$, but is far less potent against human complex III, which it inhibits with an $\mathrm{IC}_{50}$ of 70-460 nM (refs 20,21). It was previously assumed that this comparably low efficacy against purified human complex III would not translate to the inhibition of this enzyme in vitro. However, several experiments conducted in this study indicate that atovaquone inhibits complex III in cancer cells. First, the OCR is inhibited by atovaquone under conditions promoting complex III-dependent respiration in digitonin-permeabilized cells. Second, the reduction of cytochrome $c$ is inhibited by atovaquone under conditions promoting complex III-dependent respiration in isolated mitochondria, but the reduction of dichlorophenolindophenol by complex II is not inhibited by atovaquone. Furthermore, complex III inhibition by atovaquone, antimycin A or myxothiazol is sufficient to markedly reduce the OCR and consequently to eradicate spheroid hypoxia and improve radiation response.

Complex III and DHODH, an enzyme of the de novo pyrimidine synthesis pathway, are situated in the inner mitochondrial membrane in higher eukaryotes, and their activities are closely linked. Cellular DHODH activity is inhibited by ETC agonists such as antimycin A and nitric oxide, and by DHODH agonists such as leflunomide and brequinar, but only DHODH agonists inhibit the activity of purified $\mathrm{DHODH}^{24,35,36}$. Atovaquone is a much more potent inhibitor of purified human complex III $\left(\mathrm{IC}_{50}=70-460 \mathrm{nM}\right)$ than human DHODH $\left(\mathrm{IC}_{50}=14.5 \mu \mathrm{M}\right)$, suggesting that it primarily acts as a complex III inhibitor in cancer cells $s^{20-22}$. For these reasons, it is our conjecture that atovaquone primarily inhibits complex III, which causes depletion of the DHODH cofactor, ubiquinone, and subsequent inhibition of DHODH activity and downstream pyrimidine synthesis.

The anti-diabetic drug metformin, reduces the OCR by inhibition of complex I, and decreases tumour hypoxia ${ }^{11,12}$. This study demonstrated that metformin and phenformin reduce the OCR in FaDu and HCT116 cells, eliminate hypoxia in FaDu and HCT116 spheroids, and elicit an improved radiation response. In contrast, metformin and phenformin were ineffective in reducing the OCR in H1299 cells, or in alleviating H1299 spheroid hypoxia. In comparison, the efficacy of atovaquone is very similar in a wide range of tumour cell lines, and it eliminates hypoxia in $\mathrm{FaDu}$, HCT116 and H1299 spheroids.

In accordance with a previous publication ${ }^{12}$, this study demonstrated a decrease in tumour hypoxia following 7 days metformin treatment, albeit non-significant. The reason that metformin eliminated spheroid hypoxia but caused a less significant reduction in tumour hypoxia could be due to a discrepancy between the dose required to elicit this effect in spheroids and the concentration achieved in tumours. There is an ongoing debate concerning the doses of metformin used in murine cancer models, and whether they are clinically relevant ${ }^{37,38}$. Diabetics treated with the standard clinical doses of 1,500-2,500 mg per day metformin have maximum plasma levels in the range of $10-25 \mu \mathrm{M}$, and non-diabetic cancer patients receiving 1,500-2,000 mg per day have maximum plasma levels of 2.8-7 $\mu \mathrm{M}$ (refs 38,39). In comparison, the maximum plasma levels achieved in murine models following oral administration of $125-350 \mathrm{mg} \mathrm{kg}^{-1}$ per day metformin for 2 weeks are 5-47 $\mu \mathrm{M}$, with a level of metformin in the tumours of 32-200 $\mu \mathrm{M}$ (refs 37,38). At first glance, this may make the observed effects of metformin on the OCR and spheroid hypoxia at $2 \mathrm{mM}$ appear irrelevant both in vivo and in the clinic. However, several explanations have been proposed to explain this discrepancy ${ }^{12,37,38}$. These include the predicted accumulation of metformin in the mitochondria over time to achieve doses required for complex I inhibition, the effects of the tumour microenvironment, and a lower concentration of glucose, serine and glutamine in the tumour, metabolites that have been shown to cause resistance to metformin in vitro. It is possible that the dose of metformin could be raised to achieve higher plasma concentrations in cancer patients, but this may increase potentially serious adverse effects such as lactic acidosis. Indeed, phenformin was withdrawn from clinical use in the 1970s due to a high risk of lactic acidosis. This may preclude its use as an anti-cancer therapeutic, although it is possible that a good therapeutic ratio exists for phenformin at lower doses.

In contrast to metformin, the concentration of atovaquone required to reduce the OCR in vitro and to alleviate tumour hypoxia in vivo is achievable in patients. It reaches a steady-state concentration in the blood plasma of $65 \mu \mathrm{M}$ or more after 1-2 weeks administration in pneumocystis pneumonia patients at doses that are used routinely in the clinic, but reduces the OCR by up to $90 \%$ within $2 \mathrm{~h}$ (refs 32,33 ). The mean concentration of atovaquone in the mouse blood plasma after 5 days treatment was $68.6 \mu \mathrm{M}$, suggesting that doses required to reduce tumour hypoxia could be achieved without toxicity in patients.

Although atovaquone did not have a significant effect on the cell number after $4.5 \mathrm{~h}$, there was a significant decrease in the cell number after $24 \mathrm{~h}$ in monolayer cells. We do not believe that this finding effects the interpretation of any of our data or the potential clinical applicability of atovaquone because it reduces the OCR within $2 \mathrm{~h}$ without a significant effect on cell number, it does not effect the growth of spheroids or xenograft tumours in the absence of radiation, and it has an excellent clinical safety profile.

Some studies have described cycling hypoxia in tumours, characterized by periods of hypoxia followed by periods of reoxygenation, which can lead to excessive ROS production and may cause increased resistance to therapy ${ }^{40,41}$. This phenomenon is thought to occur as a result of changes in vessel perfusion and fluctuations in the flux of red blood cells within perfused vessels ${ }^{40}$. However, the contribution of tumour cells that undergo cycling hypoxia to poor patient outcome is currently unclear. Reoxygenation has also been shown to increase the potential risk of metastasis ${ }^{42,43}$. These studies highlight potential issues with reoxygenating tumours. However, the literature overwhelmingly suggests that the benefits of eradicating hypoxia outweigh any theoretical risks, with a recent meta-analysis supporting hypoxia modification as a strategy to improve response to therapy in head and neck squamous cell carcinoma ${ }^{1,3,4}$.

This study suggests that attenuation of tumour hypoxia by atovaquone may be a highly appealing clinical strategy to increase the sensitivity of tumour cells to radiation in multiple different tumour types. Accordingly, we are now initiating a proof of principle, 'window of opportunity' clinical trial to investigate whether atovaquone reduces tumour hypoxia in lung cancer patients prior to surgical resection, on the basis of imaging, serological markers and immunohistochemical markers.

\section{Methods}

Cell lines and compounds. All cell lines were purchased from the American Type Culture Collection (ATCC). STR profiling (DNA Diagnostics Centre, UK) and 
mycoplasma testing (Lonza) are conducted routinely for these cell lines. Cells were cultured at $37^{\circ} \mathrm{C}$ with $5 \% \mathrm{CO}_{2}$ in Dulbecco's modified Eagle's medium (DMEM, Sigma) supplemented with $10 \%$ fetal bovine serum (FBS, Lonza). All compounds were obtained from Sigma except duroquinol (Tokyo Chemical Industry), and EF5, a gift from Cameron Koch's Laboratory, University of Pennsylvania). All compounds were prepared from powder. For the experiments under hypoxic conditions, cells were grown at $0.5 \% \mathrm{O}_{2}$ in an in vivo 2400 Ruskinn chamber (Biotrace Fred Baker), and at $<0.1 \% \mathrm{O}_{2}$ in a Bactron II chamber (Shell Labs).

OCR primary screen. FaDu cells were incubated with either the Pharmakon 1,600 (Microsource) or the 97-compound TDI Oncology Drug Set (Target Discovery Institute, Oxford University) compound libraries at 2 and $10 \mu \mathrm{M}$ for $24 \mathrm{~h}$ in DMEM containing $5 \mathrm{mM}$ glucose and $4 \mathrm{mM}$ L-glutamine. The mitochondrial-specific OCR was determined by taking a basal OCR measurement in XF assay medium (Seahorse Biosciences) containing $5 \mathrm{mM}$ galactose, $5 \mathrm{mM}$ sodium pyruvate and $4 \mathrm{mM}$ L-glutamine using an XF96 Analyzer (Seahorse Biosciences) and subtracting the OCR measured following injection of $2 \mu \mathrm{M}$ antimycin A. The cells were then fixed in ice-cold methanol for $5 \mathrm{~min}$, and incubated in $1 \times \mathrm{PBS}, 4 \mu \mathrm{g} \mathrm{ml}^{-1}$ Hoechst 33258 (Sigma) for $30 \mathrm{~min}$ before measuring fluorescence using a POLARstar Omega plate reader (BMG Labtech) to obtain the relative cell number per well. The mitochondrial-specific OCR was corrected using the relative cell numbers, and compounds that caused a reduction in cell number of more than $66 \%$ compared with the DMSO control wells for each plate were excluded. Two repetitions of the screen were conducted. The $Z$ scores were generated and used to rank the compounds for each repeat, and the rank product was used to sort the compounds. The compounds with the top 130 rank products were chosen for the secondary screen.

OCR secondary screen. The secondary screen was conducted using the same protocol as the primary screen, except FaDu cells were incubated with $80 \mathrm{nM}$, $400 \mathrm{nM}, 2 \mu \mathrm{M}$ and $10 \mu \mathrm{M}$ of the compounds, the assay medium contained $5 \mathrm{mM}$ glucose, $5 \mathrm{mM}$ sodium pyruvate and $4 \mathrm{mM}$ L-glutamine, and three replicates were conducted. The analysis was conducted as described for the primary screen, using the OCR score to rank the compounds for each repeat. The OCR score is defined as the 'OCR normalized to cell number' - 'Average of OCR normalized to cell number for the DMSO wells for each plate'/'s.d. of OCR normalized to cell number for the DMSO wells for each plate'. The list was filtered to include only compounds approved for systemic use in humans, excluding those approved for topical or veterinary use. The rank product was used to sort the compounds. Compounds were excluded based on prospectively defined criteria: OCR, cell viability, pharmacokinetics, FDA-approved use and known hypoxia modification.

OCR. The cells were grown for $24 \mathrm{~h}$ in DMEM containing $5 \mathrm{mM}$ glucose and $4 \mathrm{mM}$ L-glutamine, and then measurements of the OCR were taken in XF assay medium (Seahorse Biosciences) containing $5 \mathrm{mM}$ glucose, $5 \mathrm{mM}$ sodium pyruvate and $4 \mathrm{mM}$ L-glutamine using an XF96 Analyzer (Seahorse Biosciences). For the kinetics experiments, measurements were collected every 15 min following injection of atovaquone. For the experiments with uridine and pyrimidine synthesis inhibitors, the cells were incubated with the compounds for 2 or $24 \mathrm{~h}$ as indicated, the medium was replaced with the assay medium also containing the compounds, and then measurements were made. The OCR measurements were corrected for cell number using Hoechst staining, as described above. For the experiments using permeabilized $\mathrm{FaDu}$ cells, $0.005 \%$ digitonin was the permeabilization agent, and the $\%$ OCR was measured within $15 \mathrm{~min}$ of its addition. Permeabilization of the plasma membrane allows the substrates respired by the mitochondria to be dictated by the added medium, and allows non-membrane permeable substrates to access the mitochondria ${ }^{44}$. The activities of complexes I and II were assayed using substrates specific to each complex in conjunction with an inhibitor of the other complex: $5 \mathrm{mM}$ pyruvate with $1 \mathrm{mM}$ malic acid to promote complex I-dependent respiration, and $10 \mathrm{mM}$ succinate with $1 \mu \mathrm{M}$ rotenone to promote complex II-dependent respiration. Complex III-dependent respiration was assayed using $500 \mu \mathrm{M}$ duroquinol as a substrate. Complex IV-dependent respiration was assayed using $500 \mu \mathrm{M} N, N, N^{\prime}, N^{\prime}$-tetramethyl-p-phenylenediamine (TMPD) as a substrate, in conjunction with $2 \mathrm{mM}$ ascorbate to maintain TMPD in reduced form. The inhibitors used for the assay were $2 \mu \mathrm{M}$ rotenone for complex I, $10 \mathrm{mM}$ malonate or $1 \mathrm{mM}$ malic acid for complex II, $2 \mu \mathrm{M}$ myxothiazol or $2 \mu \mathrm{M}$ antimycin A for complex III, and $20 \mathrm{mM}$ sodium azide for complex IV.

Spheroids. FaDu spheroids were grown using the liquid overlay technique, and HCT116 and H1299 spheroids were grown in lipidure-coated U-bottom plates (UMS Bio). Half of the medium was replaced twice per week. Spheroid hypoxia was quantified by EF5 staining, using an anti-EF5 antibody (both reagents obtained from University of Pennsylvania) ${ }^{7}$. Two hundred micromolar EF5 was added to the spheroids for $6 \mathrm{~h}$ before fixation in $4 \%$ paraformaldehyde for $24 \mathrm{~h}$, incubation in $30 \%$ sucrose for $2.5 \mathrm{~h}$, and then addition of OCT (VWR, TissueTek) for cryosectioning. Spheroid sections were incubated for $40 \mathrm{~min}$ in TNB blocking reagent (Perkin Elmer), washed for $5 \mathrm{~min}$ in $1 \times \mathrm{PBS}, 0.3 \%$ Tween 20, and then incubated overnight with $70 \mu \mathrm{l}$ of undiluted $75 \mu \mathrm{g} \mathrm{ml}^{-1}$ anti-EF5 antibody. Three washes of $45 \mathrm{~min}$ were then performed using $1 \times$ PBS, $0.3 \%$ Tween 20 before addition of DAPI vectorshield mounting medium (Vector Laboratories) and fluorescence microscopy (Nikon 90i, Nikon). Spheroid diameter was derived from the spheroid cross-sectional area measured by the Gelcount colony counter (Oxford Optronix) or Axiovert200M (Carl Zeiss MicroImaging GmbH).

Complex II/III and complex II assays. The complex II/III and complex II assays were performed according to the manufacturer's instructions (Cayman Chemicals), using either mitochondria isolated from $\mathrm{FaDu}$ cells or bovine heart mitochondria (Cayman Chemicals). The complex III assay uses the complex II substrate, succinate, to allow generation of the complex III substrate, ubiquinol, by complex II. One micromolar rotenone and $1 \mathrm{mM}$ cyanide were added to inhibit the activities of complexes I and IV, respectively, so the activities of complexes II and III were assayed. The complex II assay uses succinate as a substrate for complex II, and measures reduction of the redox dye, dichlorophenolindophenol, by the ubiquinol generated by complex II. One micromolar rotenone, $10 \mu \mathrm{M}$ antimycin $\mathrm{A}$ and $1 \mathrm{mM}$ cyanide were added to inhibit the activities of complexes I, III and IV, respectively, so the activity of complex II was assayed in isolation. For isolation of mitochondria, FaDu cells were resuspended in $1 \mathrm{ml}$ homogenization buffer at $4{ }^{\circ} \mathrm{C}: 10 \mathrm{mM}$ Tris, $0.1 \mathrm{mM}$ EDTA, $11.5 \%$ sucrose, $1 \%$ complete mini protease inhibitor cocktail (Roche), $\mathrm{pH} 7.4$ (ref. 47). The cells were snap frozen on dry ice and then thawed at $37^{\circ} \mathrm{C}$ ten times, centrifuged at $750 \mathrm{~g}$, resuspended in $500 \mu \mathrm{l}$ homogenization buffer, and centrifuged again, collecting the supernatants at each stage. Finally the pellet was resuspended in $100 \mu \mathrm{l}$ homogenization buffer omitting EDTA, and stored at $-80^{\circ} \mathrm{C}$.

High-performance liquid chromatography. To quantify NTPs, the cells were lifted and lysed in $100 \mu \mathrm{l}$ of $6 \%$ TCA (ref. 48). Nucleotides were neutralized by extracting the samples with $100 \mu \mathrm{l}$ of freon (1,1,2 trichlorotrifluorethane): trioctylamine (4:1). The aqueous upper layer was removed and then $40 \mu \mathrm{l}$ was injected. Chromatography was performed on a Waters 2695 system with diode array detection (Waters 2996), monitoring at $254 \mathrm{~nm}$, with an Ace column $(3 \mu \mathrm{m}$, $3 \times 125 \mathrm{~mm}$ ) maintained at $30^{\circ} \mathrm{C}$. The samples were maintained at $10^{\circ} \mathrm{C}$ Separation was attained with eluent A $(10 \mathrm{mM}$ potassium dihydrogen phosphate, $10 \mathrm{mM}$ tetrabutylammonium hydroxide, $10 \%$ methanol, $\mathrm{pH} 6.9$ ) and eluent $\mathrm{B}$ $(50 \mathrm{mM}$ potassium dihydrogen phosphate, $6 \mathrm{mM}$ tetrabutylammonium hydroxide, $30 \%$ methanol, $\mathrm{pH} 7$ ), using a flow rate of $0.6 \mathrm{ml} \mathrm{min}^{-1}$ and a gradient of $25-80 \% \mathrm{~B}$ over $20 \mathrm{~min}$, and a run time of $25 \mathrm{~min}$. Nucleotides were quantitated against commercially available dNTPs. Peak areas are presented relative to the DMSO control and normalized to the cell number obtained by a Sceptre cell counter (Millipore). Plasma and tumour atovaquone concentrations were determined using lapachol as an internal standard ${ }^{45}$ by negative electrospray ionization on a Waters EMD1000. HPLC was performed on a Cortecs C18 column, $100 \times 3 \mathrm{~mm}$ (Waters), with eluents of $5 \mathrm{~mm}$ ammonium formate in $50 \%$ acetonitrile (A) and acetonitrile (B) using a gradient of $10-100 \% \mathrm{~B}$ in $5 \mathrm{~min}$. The flow rate was $0.5 \mathrm{ml} \mathrm{min}^{-1}$. Plasma and tumour metformin concentrations were determined using HPLC with absorbance detection at $330 \mathrm{~nm}$ using phenformin as an internal standard ${ }^{46}$. Tumours were weighed and homogenized in four volumes of water. To $20 \mu \mathrm{l}$ plasma or tumour homogenate was added $20 \mu \mathrm{l}$ phenformin $(25 \mu \mathrm{M}), 10 \mu \mathrm{l} 50 \mathrm{mM} \mathrm{HCl}$ and $0.5 \mathrm{ml}$ acetonitrile. Samples were centrifuged and the supernatant dried down in a heated centrifugal evaporator. Samples were reconstituted in $100 \mu$ l Synergy water. HPLC was performed on a Gemini C18 column, $150 \times 3 \mathrm{~mm}, 3 \mu \mathrm{m}$ (Phenomenex) at $35^{\circ} \mathrm{C}$, with eluents of $10 \mathrm{mM}$ ammonium bicarbonate $\mathrm{pH} 10.4(\mathrm{~A})$ and acetonitrile (B) using isocratic elution of $60 \% \mathrm{~B}$ and a flow rate of $0.4 \mathrm{ml} \mathrm{min}^{-1}$.

Animal models. The project licence covering the animal work (PPL30/2922) was approved by the Oxford University Animal Welfare and Ethical Review Body (AWERB) and granted by the UK Home Office Animals in Science Regulation Unit (ASRU) under the Animals (Scientific Procedures) Act 1986 (ASPA)). FaDu $\left(1 \times 10^{6}\right)$ or HCT116 $\left(5 \times 10^{6}\right)$ cells were inoculated subcutaneously with matrigel (BD Biosciences) in athymic BALB/c nude female mice at age 55-70 days. Once the tumours had reached $100 \mathrm{~mm}^{3}$, atovaquone was administered in the drinking water at $50 \mathrm{mg} \mathrm{kg}^{-1}$ per day with $2 \%$ DMSO and $0.1 \%$ carboxymethylcellulose to reflect the clinical administration of atovaquone as an oral suspension ${ }^{18,32,33}$, assuming that a $20 \mathrm{~g}$ mouse would consume $5 \mathrm{ml}$ water per day. Mice were given this dose for 7 days because atovaquone takes 1-2 weeks to reach a steady-state plasma concentration in patients ${ }^{32,33}$. Tumours were harvested after i.p. administration of $0.01 \mathrm{ml} \mathrm{g}^{-1}$ body weight of $10 \mathrm{mM}$ EF5 after 7 days treatment. Tumours were measured with calipers. For the radiation treatments, a single dose of $6 \mathrm{~Gy}$ was given on day 7 with a $250-\mathrm{kV}$ orthovoltage irradiator (Philips RT 250) at a dose rate of $2.63 \mathrm{~Gy} \mathrm{~min}^{-1}$, using copper shielding. Necrosis was quantified by inspection of Hematoxylin and Eosin (H\&E) stained sections. Animals were randomly assigned to each treatment group on the day on which their tumours reached $100 \mathrm{~mm}^{3}$ using a random number generator (Excel), but the experiment was not blinded. A sample size of five mice per group was used and has $90 \%$ power (s.d. $=30 \%$, alpha $=0.05$ ) to detect a $70 \%$ decrease, based on our previous experience and published data ${ }^{5,7}$. A one-tailed test was performed for this determination. Use of five animals per treatment group allowed us to overcome the problem of differences in variance between groups being statistically compared One tumour treated with both atovaquone and radiation had an excellent response, reaching $500 \mathrm{~mm}^{3} 42$ days after the initiation of drug treatment, and was excluded from our analyses. 
Immunoblotting. Protein lysates were made using RIPA lysis buffer (Thermo Scientific). SDS-PAGE electrophoresis was conducted followed by immunoblotting. The primary antibodies used were anti-HIF-1 $\alpha$ (1:500, clone 54 , BD Biosciences) and anti-GAPDH (1:1,000 6C5, Novus Biologicals). The Alexa Fluor 680 secondary antibody (1:10,000, LICOR) was detected using the Odyssey imaging system (LICOR).

Colony formation assay. Cells were plated, incubated at $20 \% \mathrm{O}_{2}$ for $4 \mathrm{~h}$, and then incubated at either $20 \% \mathrm{O}_{2}$ or $<0.1 \% \mathrm{O}_{2}$ for $6 \mathrm{~h}$ with atovaquone prior to irradiation by a ceasium-137 irradiator (Gamma Service: GSR D1; dose rate $1.94 \mathrm{~Gy} \mathrm{~min}^{-1}$ ). Half an hour after irradiation, all cells were placed at $20 \% \mathrm{O}_{2}$ for a further $18 \mathrm{~h}$ before medium replacement. Colonies were stained with crystal violet and counted using the Gelcount colony counter (Oxford Optronix).

Statistics. Statistical analysis was performed with Prism 5 software (GraphPad Software Inc.). All values are presented as mean \pm s.d. except for the colony formation assays and HPLC data, which are presented as mean \pm s.e.m. Unpaired two-tailed $T$-tests were performed for the uridine and colony formation assays, and for the cell survival assays in Supplementary Fig. 1c. For all other experiments, one-way ANOVAs were performed to assess statistical significance with Bonferroni post correction. $P$ values $<0.05$ were considered significant. All in vitro experiments were repeated at least three times.

Data availability. The data supporting the findings of this study are contained within the Article and Supplementary Information files or available from the corresponding authors upon request.

\section{References}

1. Dhani, N., Fyles, A., Hedley, D. \& Milosevic, M. The clinical significance of hypoxia in human cancers. Semin. Nucl. Med. 45, 110-121 (2015).

2. Okunieff, P. et al. Angiogenesis determines blood flow, metabolism, growth rate, and ATPase kinetics of tumors growing in an irradiated bed: $31 \mathrm{P}$ and $2 \mathrm{H}$ nuclear magnetic resonance studies. Cancer Res. 51, 3289-3295 (1991).

3. Wilson, W. R. \& Hay, M. P. Targeting hypoxia in cancer therapy. Nat. Rev. Cancer 11, 393-410 (2011).

4. Overgaard, J. Hypoxic modification of radiotherapy in squamous cell carcinoma of the head and neck--a systematic review and meta-analysis. Radiother. Oncol. 100, 22-32 (2011).

5. Fokas, E. et al. Dual inhibition of the $\mathrm{PI} 3 \mathrm{~K} / \mathrm{mTOR}$ pathway increases tumor radiosensitivity by normalizing tumor vasculature. Cancer Res. 72, 239-248 (2012)

6. Grimes, D. R., Kelly, C., Bloch, K. \& Partridge, M. A method for estimating the oxygen consumption rate in multicellular tumour spheroids. J. R. Soc. Interface 11, 20131124 (2014).

7. Kelly, C. J. et al. Regulation of O consumption by the PI3K and mTOR pathways contributes to tumor hypoxia. Radiother. Oncol. 111, 72-80 (2014).

8. Secomb, T. W., Hsu, R., Ong, E. T., Gross, J. F. \& Dewhirst, M. W. Analysis of the effects of oxygen supply and demand on hypoxic fraction in tumors. Acta Oncol. (Madr). 34, 313-316 (1995).

9. Ellinghaus, P. et al. BAY 87-2243, a highly potent and selective inhibitor of hypoxia-induced gene activation has antitumor activities by inhibition of mitochondrial complex I. Cancer Med. 2, 611-624 (2013).

10. Lin, X. et al. A chemical genomics screen highlights the essential role of mitochondria in HIF-1 regulation. Proc. Natl Acad. Sci. USA 105, 174-179 (2008).

11. Wheaton, W. W. et al. Metformin inhibits mitochondrial complex I of cancer cells to reduce tumorigenesis. eLife 3, e02242 (2014).

12. Zannella, V. E. et al. Reprogramming metabolism with metformin improves tumor oxygenation and radiotherapy response. Clin. Cancer Res. 19, 6741-6750 (2013).

13. Reitzer, L. J., Wice, B. M. \& Kennell, D. Evidence that glutamine, not sugar, is the major energy source for cultured HeLa cells. J. Biol. Chem. 254, 2669-2676 (1979).

14. Lee, K. et al. Acriflavine inhibits HIF-1 dimerization, tumor growth, and vascularization. Proc. Natl Acad. Sci. USA 106, 17910-17915 (2009)

15. Khaw, M. \& Panosian, C. B. Human antiprotozoal therapy: past, present, and future. Clin. Microbiol. Rev. 8, 427-439 (1995).

16. Harada, Y., Ishii, I., Hatake, K. \& Kasahara, T. Pyrvinium pamoate inhibits proliferation of myeloma/erythroleukemia cells by suppressing mitochondrial respiratory complex I and STAT3. Cancer Lett. 319, 83-88 (2012).

17. Veal, G. J. et al. Pharmacokinetics of dactinomycin in a pediatric patient population: a United Kingdom Children's Cancer Study Group Study. Clin. Cancer Res. 11, 5893-5899 (2005).

18. Nixon, G. L. et al. Antimalarial pharmacology and therapeutics of atovaquone. J. Antimicrob. Chemother. 68, 977-985 (2013).
19. Birth, D., Kao, W. C. \& Hunte, C. Structural analysis of atovaquone-inhibited cytochrome bc1 complex reveals the molecular basis of antimalarial drug action. Nat. Commun. 5, 4029 (2014)

20. Biagini, G. A. et al. Acridinediones: selective and potent inhibitors of the malaria parasite mitochondrial bcl complex. Mol. Pharmacol. 73, 1347-1355 (2008).

21. Nilsen, A. et al. Quinolone-3-diarylethers: a new class of antimalarial drug. Sci. Transl. Med. 5, 177ra137 (2013).

22. Knecht, W., Henseling, J. \& Loffler, M. Kinetics of inhibition of human and rat dihydroorotate dehydrogenase by atovaquone, lawsone derivatives, brequinar sodium and polyporic acid. Chem. Biol. Interact. 124, 61-76 (2000).

23. Hail, Jr N., Chen, P. \& Bushman, L. R. Teriflunomide (leflunomide) promotes cytostatic, antioxidant, and apoptotic effects in transformed prostate epithelial cells: evidence supporting a role for teriflunomide in prostate cancer chemoprevention. Neoplasia 12, 464-475 (2010).

24. Loffler, M., Jockel, J., Schuster, G. \& Becker, C. Dihydroorotat-ubiquinone oxidoreductase links mitochondria in the biosynthesis of pyrimidine nucleotides. Mol. Cell. Biochem. 174, 125-129 (1997).

25. Elford, H. L. Effect of hydroxyurea on ribonucleotide reductase. Biochem. Biophys. Res. Commun. 33, 129-135 (1968).

26. Pasternak, C. A. \& Handschumacher, R. E. The biochemical activity of 6-azauridine: interference with pyrimidine metabolism in transplantable mouse tumors. J. Biol. Chem. 234, 2992-2997 (1959).

27. Overgaard, J. et al. A randomized double-blind phase III study of nimorazole as a hypoxic radiosensitizer of primary radiotherapy in supraglottic larynx and pharynx carcinoma. Results of the Danish Head and Neck Cancer Study (DAHANCA) Protocol 5-85. Radiother. Oncol. 46, 135-146 (1998).

28. Overgaard, J. et al. Five compared with six fractions per week of conventional radiotherapy of squamous-cell carcinoma of head and neck: DAHANCA 6 and 7 randomised controlled trial. Lancet 362, 933-940 (2003).

29. Sugie, C. et al. Reevaluation of the radiosensitizing effects of sanazole and nimorazole in vitro and in vivo. J. Radiat. Res. 46, 453-459 (2005).

30. Hassan Metwally, M. A., Jansen, J. A. \& Overgaard, J. Study of the population pharmacokinetic characteristics of nimorazole in head and neck cancer patients treated in the DAHANCA-5 trial. Clin. Oncol. (R. Coll. Radiol). 27, 168-175 (2015).

31. Overgaard, J., Overgaard, M. \& Timothy, A. R. Studies of the pharmacokinetic properties of nimorazole. Br. J. Cancer 48, 27-34 (1983).

32. Dixon, R., Pozniak, A. L., Watt, H. M., Rolan, P. \& Posner, J. Single-dose and steady-state pharmacokinetics of a novel microfluidized suspension of atovaquone in human immunodeficiency virus-seropositive patients. Antimicrob. Agents Chemother. 40, 556-560 (1996).

33. Falloon, J. et al. Atovaquone suspension in HIV-infected volunteers: pharmacokinetics, pharmacodynamics, and TMP-SMX interaction study. Pharmacotherapy 19, 1050-1056 (1999).

34. Rolan, P. E., Mercer, A. J., Tate, E., Benjamin, I. \& Posner, J. Disposition of atovaquone in humans. Antimicrob. Agents Chemother. 41, 1319-1321 (1997)

35. Beuneu, C. et al. Indirect inhibition of mitochondrial dihydroorotate dehydrogenase activity by nitric oxide. Free Radic. Biol. Med. 28, 1206-1213 (2000).

36. Khutornenko, A. A. et al. Pyrimidine biosynthesis links mitochondrial respiration to the p53 pathway. Proc. Natl Acad. Sci. USA 107, 12828-12833 (2010).

37. Chandel, N. S. et al. Are metformin doses used in murine cancer models clinically relevant? Cell Metab. 23, 569-570 (2016).

38. Dowling, R. J. et al. Metformin pharmacokinetics in mouse tumors: implications for human therapy. Cell Metab. 23, 567-568 (2016).

39. Graham, G. G. et al. Clinical pharmacokinetics of metformin. Clin. Pharmacokinet. 50, 81-98 (2011).

40. Kimura, H. et al. Fluctuations in red cell flux in tumor microvessels can lead to transient hypoxia and reoxygenation in tumor parenchyma. Cancer Res. 56, 5522-5528 (1996).

41. Koritzinsky, M. \& Wouters, B. G. The roles of reactive oxygen species and autophagy in mediating the tolerance of tumor cells to cycling hypoxia. Semin. Radiat. Oncol. 23, 252-261 (2013).

42. Miao, Z. F. et al. Influence of different hypoxia models on metastatic potential of SGC-7901 gastric cancer cells. Tumour Biol. 35, 6801-6808 (2014).

43. Okajima, M. et al. Anoxia/reoxygenation induces epithelial-mesenchymal transition in human colon cancer cell lines. Oncol. Rep. 29, 2311-2317 (2013).

44. Salabei, J. K., Gibb, A. A. \& Hill, B. G. Comprehensive measurement of respiratory activity in permeabilized cells using extracellular flux analysis. Nat. Protoc. 9, 421-438 (2014).

45. Gurule, S., Goswami, D., Khuroo, A. H. \& Monif, T. LC-APCI mass spectrometric method development and validation for the determination of atovaquone in human plasma. Biomed. Chromatogr. 24, 497-505 (2010).

46. Porta, V. et al. HPLC-UV determination of metformin in human plasma for application in pharmacokinetics and bioequivalence studies. J. Pharm. Biomed Anal. 46, 143-147 (2008).

47. Chaiyarit, S. \& Thongboonkerd, V. Comparative analyses of cell disruption methods for mitochondrial isolation in high-throughput proteomics study. Anal. Biochem. 394, 249-258 (2009). 
48. Moss, J. et al. Break-induced ATR and Ddb1-Cul4(Cdt)(2) ubiquitin ligase-dependent nucleotide synthesis promotes homologous recombination repair in fission yeast. Genes Dev. 24, 2705-2716 (2010).

\section{Acknowledgements}

We thank Graham Brown, Lucy Sansom, Daniel V. Ebner and Alison Howarth for technical assistance. EF5 (2-(2-Nitro-1H-imidazol-1-yl)- $N$-(2,2,3,3,3-pentafluoropropyl) acetamide) was a gift from the laboratory of Cameron Koch. The funding sources were Cancer Research UK, Medical Research Council and National Institute for Health Research Biomedical Research Centre, Oxford. G.S.H. is supported by a Cancer Research UK Clinician Scientist Award (Grant number C34326/A13092).

\section{Author contributions}

T.M.A. designed, executed and analysed all experiments with the following exceptions: E.F. and T.M.A. conducted the in vivo experiments. S.A. contributed to the experiments in hypoxic conditions. L.K.F. and M.S. performed the HPLC. M.S. formulated the atovaquone for the in vivo studies. M.H. performed the spheroid regrowth experiments and T.M.A. analysed the data. G.P. contributed to the analysis of the spheroid data. C.J.K. and F.M.B. advised on the screen design and analysis, respectively. G.S.H., W.G.McK. and R.J.M. supervised the project. T.M.A. wrote the article with advice from G.S.H., W.G.McK., R.J.M., L.A.K.-S. and E.M.H

\section{Additional information}

Supplementary Information accompanies this paper at http://www.nature.com/ naturecommunications

Competing financial interests: The authors declare no competing financial interests.

Reprints and permission information is available online at http://npg.nature.com/ reprintsandpermissions/

How to cite this article: Ashton, T. M. et al. The anti-malarial atovaquone increases radiosensitivity by alleviating tumour hypoxia. Nat. Commun. 7:12308 doi: $10.1038 /$ ncomms12308 (2016).

This work is licensed under a Creative Commons Attribution 4.0 International License. The images or other third party material in this article are included in the article's Creative Commons license, unless indicated otherwise in the credit line; if the material is not included under the Creative Commons license, users will need to obtain permission from the license holder to reproduce the material. To view a copy of this license, visit http://creativecommons.org/licenses/by/4.0/

(C) The Author(s) 2016 